NASA Technical Memorandum 102469

AIAA-90-0643

\title{
Analysis of Rotary Engine Combustion Processes Based on Unsteady, Three-Dimensional Computations
}

M.S. Raju

Sverdrup Technology, Inc.

NASA Lewis Research Center Group

Cleveland, Ohio

and

E.A. Willis

National Aeronautics and Space Administration

Lewis Research Center

Cleveland, Ohio

Prepared for the

28th Aerospace Sciences Meeting sponsored by the American Institute of Aeronautics and Astronautics Reno, Nevada, January $8-\overline{11}, \overline{1990}$

\section{NASA}

(NASA-IM-102469) ANALYSIS OF ROTARY ENGINE

N9O-13749 COMPUSTION PRTCESSES BASEO ON UNSTEADY, 
Analysis of Rotary Engine Combustion Processes Based on Unsteady, Three-Dimensional Computations

\author{
M. S. Raju ${ }^{\dagger}$ \\ Sverdrup Tech., Inc., NASA Lewis Research Center,Cleveland, Ohio \\ E.A. Willis $\ddagger$ \\ NASA Lewis Research Center, Cleveland, Ohio
}

\begin{abstract}
A new computer code has been developed for predicting the turbulent, and chemically reacting flows with sprays occurring inside of a stratified-charge rotary engine (SCRE). The solution procedure is based on an Eulerian-Lagrangian approach where the unsteady, three-dimensional Navier-Stokes equations for a perfect gas-mixture with variable properties are solved in generalized, Eulerian coordinates on a moving grid by making use of an implicit finite-volume, Steger-Warming flux vector splitting scheme, and the liquid-phase equations are solved in Lagrangian coordinates. Both the details of the numerical algorithm and the finite-difference predictions of the combustor flowfield during the opening of exhaust and/or intake, and also during fuel vaporization and combustion, are presented.
\end{abstract}

\title{
I. Introduction
}

The rotary combustion engine (RCE) would be desirable as a powerplant for light aircraft, drones (including high-altitude application), auxiliary and ground power units, and also for marine and industrial application, if only its efficiency could be improved closer to that of diesel engines. It has inherent advantages over reciprocating engines in terms of higher airflow capacity, higher power-to-weight ratio, and less vibration, among others. An initial attempt to introduce a gasoline-fueled rotary engine into the general aviation market was unsuccessful because of poor fuel economy, uncertain availability of avgas, and marginal weight advantage over contemporary reciprocating engines. Subsequent research sponsored by industry, NASA, and the Navy has led to the development of the stratified-charge rotary engine (SCRE) concept, which has demonstrated multifuel capability. Current R\&D sponsored by NASA is aimed at reducing the cruise brake specific fuel consumption (BSFC, lb/bhp-hr) from a current value of about 0.42 to 0.35 or less by the end of 1992. The expected improvement will be enabled by further CFD - driven fuel injection,

\footnotetext{
$\dagger_{\text {Senior Research Engineer }}$

$\ddagger_{\text {Chief Engineer, Propulsion System Division, Member AIAA }}$
} 
spray, and nozzle optimizations, rotor pocket and nozzle relocations, and related modifications. In this paper, we address the CFD aspect of the technology development program for predicting the complex flow patterns occurring inside of a Wankel engine.

Early modelling efforts on the Wankel engine were based on thermodynamic models ${ }^{1,2}$ and also on one-dimensional modelling of premixed-charge combustion. ${ }^{3}$ Multi-dimensional models of the Wankel engine are very recent in origin; Grasso et al. ${ }^{4}$ have presented the first three-dimensional computations of a SCRE during the early stages of flame propagation. Subsequent computations performed by Abraham and $\mathrm{Bracco}^{5,6}$ have led to some important design changes in the rotary engine development at John Deere \& Co., especially in the fuel injector configuration. Their code, REC-3D-FSC-86, is a modifed version of the KIVA code developed at Los Alamos National Laboratory $^{7}$ for the modelling of reciprocating engines. KIVA makes use of a conditionally stable algorithm, and the stability of the KIVA scheme is improved by making use of an acoustic subcycling step in order to alleviate the stiffness problems arising from compressibility effects. There appears to be considerable room for improvement in the code, since it neglects the spatial gradients whenever the grid spacing becomes smaller than some predefined value and also requires excessive CPU time when the engine speed becomes small. Shih et al. ${ }^{8}$ presented the first two-dimensional computations of a motored Wankel engine in the absence of combustion. Their code, LEWIS-2D, is based on the Beam-Warming type of ADI method. Their computations have subsequently been extended to three dimensions in Steinthorsson et al..$^{9}$ Linear stability analysis has shown that the ADI method is unconditionally stable in two dimensions but is unconditionally unstable in three dimensions. Although artificial dissipation has some stabilizing effect, an excessive amount can impair stability and reduce accuracy and convergence. Recently, Li et al. ${ }^{10}$ have modified their LEWIS-3D code based on upwind schemes together with the incorporation of a $k-\epsilon$ turbulence model.

The present solution procedure differs from both REC-3D-FSC-86 and LEWIS-3D in many ways in terms of the numerics, and also the submodels used for turbulence, combustion, and sprays.

\section{Physical Description}

A schematic of the Wankel engine is shown in Fig. 1. The Wankel engine is composed of a peripheral housing with provisions for the intake and exhaust ports, fuel injector and spark igniter, a three-flank rotor, and a crank shaft. The contour of the inner surface of the outer casing of the Wankel engine is composed of a two-lobe peritrochoid. ${ }^{11}$ The contour of a rotor revolving along an outer housing is represented by a peritrochoid inner envelope. The geometric analysis of the rotor and housing surfaces can be found in Yamamoto. 11 The rotor surface is further modified by the formation of a rotor pocket. The presence of a rotor pocket not only alters the expansion and/or compression ratio of the engine but also plays an important role in modifying the flowfield, and mixing and combustion characteristics of the combustor. The present rotor configuration is adopted from Steinthorsson et al. ${ }^{9}$ 
The rotor turns eccentrically at one third of the crank shaft speed. The three combustion chambers of the Wankel engine are the three regions enclosed between the three rotor faces and the peritrochoid housing, two side housings, two side seals, and lead and trail apex seals. In the present calculations, only one of the three combustion chambers is considered, since leakage through the seals is assumed to be negligible. As the rotor revolves around the crank shaft, each of the combustion chambers is continually deformed. This produces the necessary compression and expansion of the fluid for the required engine performance during each one of the cyclic operations. The intake and exhaust ports, spark igniter, and the fuel injector are located along the peritrochoid housing as shown in Fig. 1.

In the present study, the computations are initiated before the opening of the exhaust port for the combustor formed with the second rotor flank as shown in Fig. 1. The initial conditions correspond to the conditions of quiescent air at pressure, $P_{\text {in }}=1 \mathrm{~atm}$, and temperature, $T_{\text {in }}$ $=300 \mathrm{~K}$. As the rotor moves in the clockwise direction the exhaust port opens and the residual gas moves out of the combustion chamber, since the normally imposed pressure in the exhaust remains lower than the interior engine pressure during most of the compression cycle. The exhaust conditions are given by

$$
\begin{array}{r}
\frac{\partial \rho}{\partial n}=\frac{\partial y_{i}}{\partial n}=\frac{\partial e}{\partial n}=0 \\
P=P_{e x h}, u=w=0 \\
v=-C_{d c}\left[\frac{2\left(P-P_{e x h}\right)}{\rho}\right]^{0.5}
\end{array}
$$

where $\rho, e$, and $y$ are the fluid density, internal energy, and mass fraction, respectively; $u, v$, and $w$ are the velocity components in Cartesian coordinates; $C_{d c}(=0.9)$ is the discharge coefficient; and subscript $i$ and $n$ represent species and the normal component of the boundaries, respectively.

As the rotor moves further in the clockwise direction, the intake port opens and fresh air moves into the combustion chamber. There is an overlapping region during which both the exhaust and intake ports are simultaneously open before the exhaust port closes completely. During this process not only the residual gas but also some of the fresh intake might escape through the exhaust port. Most of the intake occurs during the expansion stroke of the engine. The inport conditions are given by

$$
\begin{array}{r}
P=P_{\text {int }}, T=T_{\text {int }}, y_{i}=y_{i, i n t}, \\
\rho=\rho_{\text {int }}, u=w=0 \\
v=-C_{d c}\left[\frac{2\left(P_{\text {int }}-P\right)}{\rho}\right]^{0.5}
\end{array}
$$


As the rotor turns further, the intake port closes and the liquid fuel is injected into the chamber during the compression stroke before the top-dead center (TDC) is reached. Spark injection provides the initial energy needed for the early droplet evaporation and also helps to promote ignition of the vaporized fuel and air mixture. Most of the combustion is completed as the rotor moves past the TDC, and most of the residual combustion products are eventually driven out of the combustion chamber through the exhaust port. The whole combustion performance is determined by a very complex interaction of various engine parameters including the location of the exhaust and intake ports, shape of the rotor pocket, injector and spark timings, fuel properties, and many others.

\section{Gas-Phase Equations in Generalized Coordinates}

The governing unsteady equations based on the conservation of mass, momentum, energy, and species for turbulent, reacting, and compressible flows are presented in strong conservation law form. The exchanges of mass, momentum, and energy through liquid-phase interaction are considered by the inclusion of appropriate source terms. The Reynolds-averaged equations are formulated in generalized coordinates to accommodate the time-variation of the complex combustor geometry.

$$
\begin{gathered}
\frac{\partial \vec{Q}}{\partial t}+\frac{\partial \vec{F}}{\partial \xi}+\frac{\partial \vec{G}}{\partial \eta}+\frac{\partial \vec{H}}{\partial \xi}=\frac{\partial \vec{F}_{v}}{\partial \xi} \\
+\frac{\partial \vec{G}_{v}}{\partial \eta}+\frac{\partial \vec{H}_{v}}{\partial \zeta}+\vec{S}_{l}+\vec{S}_{c}
\end{gathered}
$$

where

$$
\vec{Q}=\left(\begin{array}{c}
\rho D \\
\rho D u \\
\rho D v \\
\rho D w \\
\rho D e \\
\rho D y_{f} \\
\rho D y_{0}
\end{array}\right) \quad \vec{F}=\left(\begin{array}{c}
\rho D\left(U+\xi_{t}\right) \\
\rho D u\left(U+\xi_{t}\right)+P D \xi_{x} \\
\rho D v\left(U+\xi_{t}\right)+P D \xi_{v} \\
\rho D w\left(U+\xi_{t}\right)+P D \xi_{x} \\
\rho D e\left(U+\xi_{t}\right)+P D U \\
\rho D y_{f}\left(U+\xi_{t}\right) \\
\rho D y_{0}\left(U+\xi_{t}\right)
\end{array}\right)
$$




$$
\begin{aligned}
& \vec{G}=\left(\begin{array}{c}
\rho D\left(V+\eta_{t}\right) \\
\rho D u\left(V+\eta_{t}\right)+P D \eta_{z} \\
\rho D v\left(V+\eta_{t}\right)+P D \eta_{v} \\
\rho D w\left(V+\eta_{t}\right)+P D \eta_{z} \\
\rho D e\left(V+\eta_{t}\right)+P D V \\
\rho y_{f}\left(V+\eta_{t}\right) \\
\rho D y_{0}\left(V+\eta_{t}\right)
\end{array}\right) \\
& \vec{H}=\left(\begin{array}{c}
\rho D\left(W+s_{t}\right) \\
\rho D u\left(W+s_{t}\right)+P D s_{x} \\
\rho D v\left(W+s_{t}\right)+P D s_{y} \\
\rho D w\left(W+s_{t}\right)+P D s_{z} \\
\rho D e\left(v+s_{t}\right)+P D W \\
\rho D y_{f}\left(W+s_{t}\right) \\
\rho D y_{0}\left(W+s_{t}\right)
\end{array}\right) \\
& \vec{F}_{v}=\left(\begin{array}{c}
f_{v 1} \\
f_{v 2} \\
f_{v 3} \\
f_{v 4} \\
f_{v 5} \\
f_{v 6} \\
f_{v 7}
\end{array}\right)=\left(\begin{array}{c}
0 \\
D \xi_{x} \tau_{x x}+D \xi_{y} \tau_{x y}+D \xi_{x} \tau_{x z} \\
D \xi_{x} \tau_{x y}+D \xi_{y} \tau_{v y}+D \xi_{z} \tau_{y z} \\
D \xi_{x} \tau_{z z}+D \xi_{y} \tau_{y z}+D \xi_{z} \tau_{z z} \\
u f_{v 2}+v f_{v 3}+w f_{v 4}+k\left(C_{1} \frac{\partial T}{\partial \xi}+C_{2} \frac{\partial T}{\partial \eta}+C_{3} \frac{\partial T}{\partial \zeta}\right) \\
\rho D_{f m}\left(C_{1} \frac{\partial v f}{\partial \xi}+C_{2} \frac{\partial v t}{\partial \eta}+C_{3} \frac{\partial v_{1}}{\partial \zeta}\right) \\
\rho D_{v m}\left(C_{1} \frac{\partial v_{0}}{\varepsilon \xi}+C_{2} \frac{\partial v_{0}}{\delta \eta}+C_{3} \frac{\partial y_{0}}{\partial s}\right)
\end{array}\right)
\end{aligned}
$$

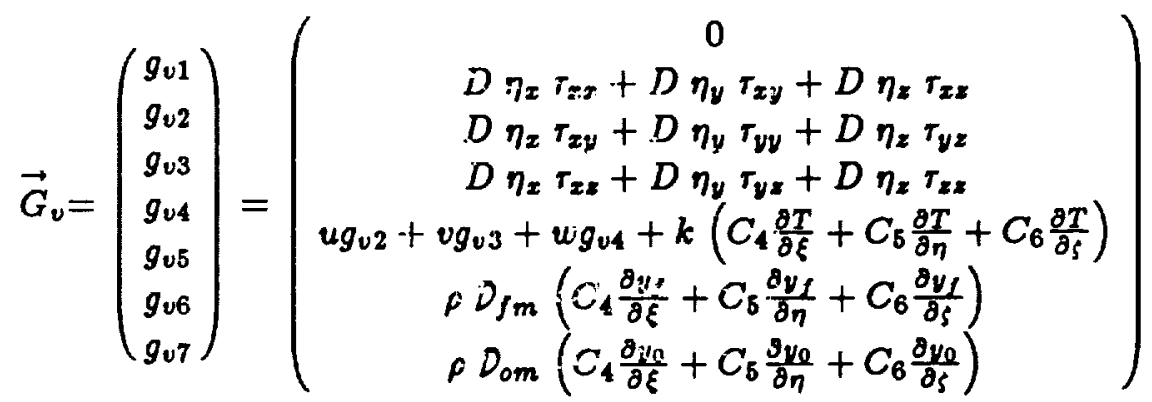




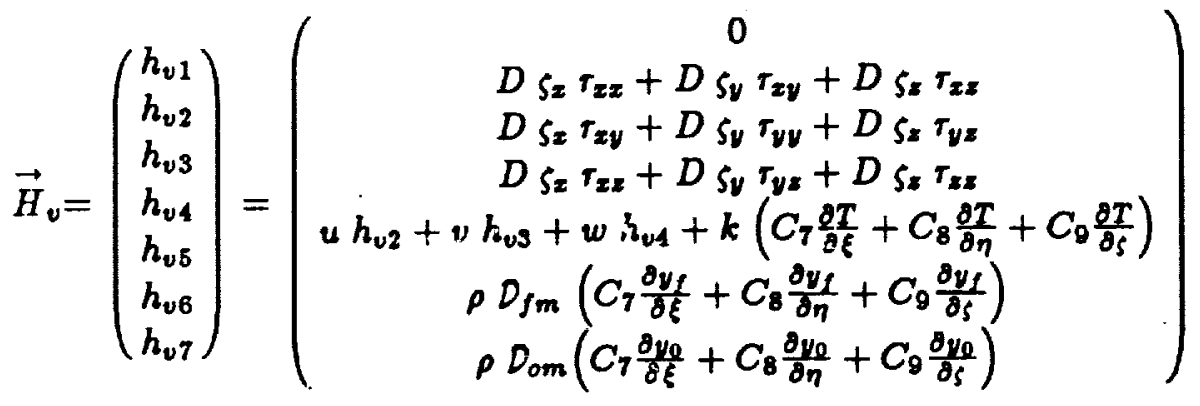

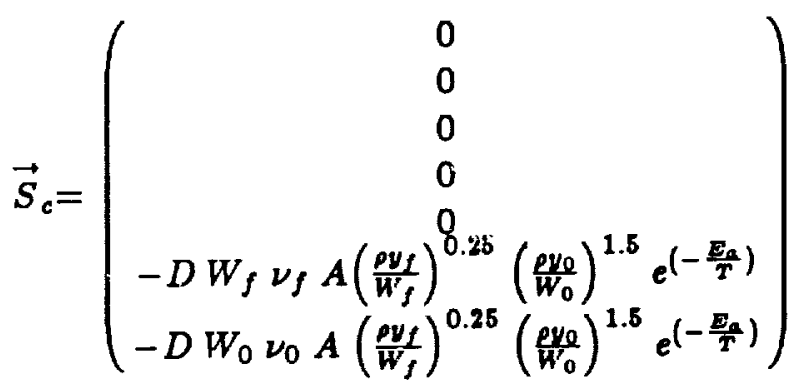

$$
\begin{aligned}
& \vec{S}_{1}=\left(\begin{array}{c}
\sum_{k} n_{k} m_{k} \\
\sum_{k} n_{k} m_{k} u_{k}-\frac{4 \pi}{3} \rho_{k} r_{k}^{3} n_{k} \frac{d u_{k}}{d t} \\
\sum_{k} n_{k} m_{k} v_{k}-\frac{4 \pi}{3} \rho_{k} r_{k}^{3} n_{k} \frac{d v_{k}}{d t} \\
\sum_{k} n_{k} m_{k} w_{k}-\frac{1 \pi}{3} \rho_{k} r_{k}^{3} n_{k} \frac{d w_{k}}{d t} \\
\sum_{k} n_{k} m_{k}\left(h_{j \iota}-l_{k, e f f}\right) \\
\sum_{k} n_{k} m_{k} \\
0
\end{array}\right) \\
& C_{1}=D\left(\xi_{x}^{2}+\xi_{y}^{2}+\xi_{z}^{2}\right) \\
& C_{2}=D\left(\xi_{x} \eta_{x}+\xi_{y} \eta_{y}+\xi_{z} \eta_{z}\right) \\
& C_{3}=D\left(\xi_{z} \zeta_{x}+\xi_{y} \zeta_{y}+\xi_{z} \zeta_{z}\right) \\
& C_{4}=D\left(\xi_{x} \eta_{z}+\xi_{y} \eta_{y}+\xi_{z} \eta_{z}\right) \\
& C_{5}=D\left(\eta_{x}^{2}+\eta_{y}^{2}+\eta_{x}^{2}\right) \\
& C_{6}=D\left(\eta_{x} \zeta_{x}+\eta_{y} s_{y}+\eta_{x} s_{z}\right) \\
& C_{y}=D\left(\xi_{x} \zeta_{x}+\xi_{y} \zeta_{y}+\xi_{z} \zeta_{z}\right)
\end{aligned}
$$




$$
\begin{aligned}
C_{8} & =D\left(\eta_{z} \zeta_{z}+\eta_{y} \zeta_{y}+\eta_{z} \zeta_{z}\right) \\
C_{9} & =D\left(\zeta_{x}^{2}+\zeta_{y}^{2}+\zeta_{z}^{2}\right) \\
\tau_{x x} & =\frac{2}{3} \mu\left(2 \frac{\partial u}{\partial x}-\frac{\partial v}{\partial y}-\frac{\partial w}{\partial z}\right) \\
\tau_{x y} & =\mu\left(\frac{\partial u}{\partial y}+\frac{\partial v}{\partial x}\right) \\
\tau_{x z} & =\mu\left(\frac{\partial u}{\partial z}+\frac{\partial w}{\partial x}\right) \\
\tau_{y y} & =\frac{2}{3} \mu\left(2 \frac{\partial v}{\partial y}-\frac{\partial u}{\partial x}-\frac{\partial w}{\partial z}\right) \\
\tau_{y z} & =\mu\left(\frac{\partial v}{\partial z}+\frac{\partial w}{\partial y}\right) \\
\tau_{z z} & =\frac{2}{3} \mu\left(2 \frac{\partial w}{\partial z}-\frac{\partial u}{\partial x}-\frac{\partial v}{\partial y}\right) \\
\mu & =\mu \mu_{l m}+\mu_{t} \\
k & =k_{l m}+\frac{\mu C_{p m}}{\operatorname{Pr}_{t}} \\
D_{i m} & =\frac{\mu}{S c_{i} \rho}
\end{aligned}
$$

and $x, y$, and $z$ are the Cartesian coordinates in the physical space; $\xi, \eta$, and $\zeta$ are the coordinates in the computational space; $D$ is the determinant of the matrix, $J$ in Eq. (6), and is also a measure of the volume of a computational cell; $y_{i}$ is the mass fraction of the $i$ th species; $\vec{S}_{c}$ is a vector representing the source terms arising from the finite-rate chemical reactions; $W_{i}$ is the Inolecular weight of the species; $\nu_{i}$ is the etoichiometric ratio of the $i$ th species participating in a given reaction step; $A$ and $E_{a}$ are the pre-exponential coefficient and activation energy of a given Arrhenius reaction-rate term; $\vec{S}_{l}$ is a vector representing the source terms arising from the liquid-phase interaction; $n_{k}$ is the number of droplets in a $k$ th characteristic representing a group of droplets; $m_{k}$ is the vaporization rate of a droplet belonging to the kth characteristic; $r_{k}$ is the droplet radius; $h_{f}$, and $l_{k, e f f}$ are the enthalpy of the fuel vapour at the droplet surface, and the effective latent heat of vaporization; $\mu_{t}$ is the turbulent viscosity; $k_{l m}$ and $\mu_{\ell m}$ are the thermal conductivity and laminar viscosity of the gas mixture and are determined using Wilke's mixing rule with fourth-order polynomial fits based upon temperature dependence ${ }^{12} ; C_{p m}$ is the specific heat of the gas mixture at constant pressure and is also determined from fourth-order polynomial fits involving temperature dependence; $P r_{t}(=0.90)$ is the turbulent Prandtl number; $S c_{t}(=0.90)$ is the turbulent Schmidt number; the subscripts $f, o, l, \ell, c, m$, and $k$ represent fuel, oxidizer, liquid-phase, laminar, chemical reaction, gasecus mixture, and characteristic, respectively.

The pressure and temperature are calculated iteratively from the following procedure: 


$$
e=\sum_{i=1}^{N_{i}} y_{i} h_{i}-\frac{P}{\rho}+\frac{1}{2}\left(u^{2}+v^{2}+w^{2}\right)
$$

where

$$
\begin{gathered}
h_{i}=h_{f i}^{0}+\int_{T_{\text {ref }}}^{T} C_{p i} y_{i} d T \\
C_{p i}=\frac{R_{u}}{W_{i}}\left(A_{1 i}+A_{2 i} T+A_{3 i} T^{2}+A_{4 i} T^{3}+A_{5 i} T^{4}\right) \\
P=\rho R_{u} T \sum_{i=1}^{N_{i}} \frac{y_{i}}{W_{i}}
\end{gathered}
$$

where $h_{f i}^{o}$ is the heat of formation of ith species, and $R_{u}$ is the universal gas constant. Equation (5) is the equation of state for a gas mixture of $H$, species. The Jacobians of the coordinate transformation are given by

$$
J=\left(\begin{array}{lll}
x_{\xi} & x_{\eta} & x_{\xi} \\
y_{\xi} & y_{\eta} & y_{\xi} \\
z_{\xi} & z_{\eta} & z_{\xi}
\end{array}\right)
$$

and

$$
J^{-1}=\left(\begin{array}{ccc}
\xi_{x} & \xi_{y} & \xi_{x} \\
\eta_{x} & \eta_{y} & \eta_{x} \\
\zeta_{x} & \zeta_{y} & \zeta_{z}
\end{array}\right)
$$

The metric coefficients resulting from the coordinate transformation are evaluated from the following identities:

$$
\begin{aligned}
& \xi_{z}=\frac{1}{D}\left(\left(y_{\eta} z\right)_{:}-\left(y_{\zeta} z\right)_{\eta}\right) \\
& \xi_{y}=\frac{1}{D}\left(\left(z_{\eta} x\right)_{s}-\left(z_{\zeta} x\right)_{\eta}\right) \\
& \xi_{z}=\frac{1}{D}\left(\left(x_{\eta} y\right)_{s}-\left(z_{\zeta} y\right)_{\eta}\right) \\
& \eta_{z}=\frac{1}{D}\left(\left(y_{\zeta} z\right)_{\xi}-\left(y_{\xi} z\right)_{\zeta}\right) \\
& \eta_{y}=\frac{1}{D}\left(\left(z_{\zeta} x\right)_{\xi}-\left(z_{\xi} x\right)_{\zeta}\right)
\end{aligned}
$$




$$
\begin{aligned}
\eta_{z} & =\frac{1}{D}\left(\left(x_{\zeta} y\right)_{\xi}-\left(x_{\xi} y\right)_{\zeta}\right) \\
\zeta_{x} & =\frac{1}{D}\left(\left(y_{\xi} z\right)_{\eta}-\left(y_{\eta} z\right)_{\xi}\right) \\
\zeta_{y} & =\frac{1}{D}\left(\left(z_{\xi}\right)_{\eta}-\left(z_{\eta} x\right)_{\xi}\right) \\
\zeta_{z} & =\frac{1}{D}\left(\left(x_{\xi} y\right)_{\eta}-\left(x_{\eta} y\right)_{\xi}\right) \\
\xi_{t} & =-\left(\xi_{x} x_{t}+\xi_{y} y_{t}+\xi_{x} z_{t}\right) \\
\eta_{t} & =-\left(\eta_{x} x_{t}+\eta_{y} y_{t}+\eta_{z} z_{t}\right) \\
\zeta_{t} & =-\left(\varsigma_{x} x_{t}+\zeta_{y} y_{t}+\zeta_{z} z_{t}\right)
\end{aligned}
$$

It is noteworthy that the following equations represent the metric invariant terms arising from the coordinate transformation:

$$
\begin{gathered}
D_{t}+\left(D \xi_{i}\right)_{\xi}+\left(D \eta_{t}\right)_{\eta}+\left(D_{\zeta_{t}}\right)_{s}=0 \\
\left(D \xi_{x}\right)_{\xi}+\left(D \eta_{x}\right)_{\eta}+\left(D D_{\zeta_{x}}\right)_{s}=0 \\
\left(D \xi_{\nu}\right)_{\xi}+\left(D \eta_{\nu}\right)_{\eta}+\left(D S_{\zeta_{y}}\right)_{s}=0 \\
\left(D \xi_{z}\right)_{\xi}+\left(D \eta_{z}\right)_{\eta}+\left(D D_{\zeta_{z}}\right)_{s}=0
\end{gathered}
$$

When the governing equations are formulated in strong conservation form, it is essential that the left-hand side of Eqs. (9) to (12) vanish identically when the derivatives are approximated by finite-differences; otherwise spurious source terms may result from geometrically induced errors. ${ }^{13}$ Equations (10) to (12) are satisfied identically when central differences are used to evaluate the spatial derivatives. This is true since the metric identities in Eq. (8) are written in conservative form. However, the determinant of the coordinate transformation is computed numerically from the solution of $\mathrm{Eq}$. (9) in order to avoid grid-motion induced errors. ${ }^{13}$

\section{Details of the Spray, Combustion, and Turbulence Models}

Here we provide a brief description of the spray model as it is adopted from Raju and Sirignano. ${ }^{14,15}$ The solution of the liquid-phase equations is extended further in the present study from the two-dimensional to three-dimensional computations. Also, several modifications are incorporated into the interpolation procedure between the Eulerian and Lagrangian coordinate systems as the gas-phase computations are performed in the generalized coordinates as opposed to the Cartesian coordinates used in Raju et al. ${ }^{14,15}$ The interaction between the two phases is taken into account by the following procedure: (1) In order to obtain the solution of the liquid-phase 
equations, it is first necessary to know the gas-phase properties at the particle locations. In the present computations, the gas-phase properties are evaluated by using a second-order accurate interpolation method involving volume-weighted averaging; (2) The ordinary differential equations describing particle size, position, and velocity are solved by the second-order accurate Runge-Kutta method. The partial differential equation describing the transient temperature variation within the droplet interior is based on a simplified vortex model and is solved by an implicit method. The formulation for the droplet vaporization rate is based either on a simplified gas-phase boundary layer analysis or on a simplified correlation, ${ }^{15}$ depending upon the droplet Reynolds number; (3) Finally, after the liquid-phase equations are solved, the source terms evaluated at the particle location are redistributed amongst the eight computational nodes surrounding the particle by using volume-weighted averaging.

The success of the spray model also depends a great deal on the correct specification of the injector exit conditions. The injector is an eight-holes configuration and is located along the middle of the peritrochoid housing as shown in Fig. 1. 'The timing of the injector opening and closing is determined by the given engine operating conditions. The fuel emerges in a fan shape consisting of eight streams as shown in Figs. 5 and 6. Both the initial droplet velocities and temperatures are assumed to be known, and the droplet sizes are determined by the Rosen-Ramler distribution. ${ }^{16}$ The droplet injection timing is determined by the resolution of the computational cells used in the gas-phase computations. ${ }^{14}$ The present model does not take into account details of the liquid filament breakup and its subsequent effect on the conditions at the injector exit.

The solution procedure could perhaps be improved with the consideration of droplet dispersion due to turbulence. However, the effects of turbulent dispersion in the modelling of combusting sprays were found to be small in a previous study, in comparison to the uncertainties in the specification of the initial conditions at the injector exit. ${ }^{17}$ The present model is based on a dilute spray approximation where the spray characteristics are based on an isolated droplet behaviour. O'Rourke and Bracco, ${ }^{18}$ Greenberg and Tambour, ${ }^{19}$ and Asheim et al. ${ }^{20}$ have modelled liquid sprays including droplet collisions. The importance of droplet collision and breakup in the overall spray behaviour is not well established, especially in the regions of the spray where the droplet loading is low. In the present computations, the effect of variable properties in the liquid-phase is not considered, though this factor becomes very important when the droplets vaporize near the critical conditions. ${ }^{21,22}$

The combustion model is based on an analogous treatment of laminar diffusion flames with the assumption that no envelope flame exists. The reaction rate is determined based on a single-global kinetic mechanism of Westbrook and Dryer ${ }^{23}$ For $n$-decane, the kinetic mechanism is given by

$$
\mathrm{C}_{10} \mathrm{H}_{22}+15.5\left(\mathrm{O}_{2}+3.76 \mathrm{~N}_{2}\right) \rightarrow 10 \mathrm{CO}_{2}+11 \mathrm{H}_{2} \mathrm{O}+58.28 \mathrm{~N}_{2}
$$

By assuming equal binary diffusivities for all the species in the mixture, the concentrations of $\mathrm{N}_{2}, \mathrm{CO}_{2}$, and $\mathrm{H}_{2} \mathrm{O}$ can be determined from simple algebraic relationships based on the atomic 
balance of the constituent species, once the mass fractions of fuel and oxidizer are known from the solution of the two gas-phase equations based on the conservation of fuel and oxidizer.

$$
\begin{gathered}
y_{H_{2} O}=K_{2}-K_{1} K_{2} y_{O_{2}}-K_{2} y_{C_{10} H_{22}} \\
y_{C O_{2}}=K_{2} K_{3}-K_{1} K_{2} K_{3} y_{2}-K_{2} K_{3} y_{C_{10} H_{22}} \\
y_{N_{2}}=1-K_{2}-K_{2} K_{3}-y_{O_{2}}\left(1-K_{1} K_{2}-K_{1} K_{2} K_{3}\right)-y_{C_{10} H_{22}}\left(1-K_{2}-K_{2} K_{3}\right)
\end{gathered}
$$

where $K_{1}=4.29, K_{2}=0.08723$, and $K_{3}=2.222$. Note that the effect of turbulence on the reaction rate can be very important, but is not considered in the present solution procedure since a realistic model is not currently available. In the near future, we are planning to implement the turbulence-reaction model used in Raju and Sirignano ${ }^{14,15}$ which is based on the eddy break-up model of Spalding ${ }^{24}$ requiring the solution of an additional equation involving the square of fuel concentration fluctuations. The eddy break-up model provided some useful results in the modelling of premixed flames, however, its applicability in a spray environment is uncertain.

The turbulence model used is a constant eddy viscosity model of Steinthorson et al. ${ }^{8}$ where the turbulent diffusivity is given by

$$
\mu_{t}=a_{T} \Omega \rho
$$

and $a_{T}$ is a function of the crank angle $\theta$, and $\Omega$ is the crank speed. One obvious discrepancy of this model is its failure to satisfy the condition of $\mu_{t}=0$ at the walls. ${ }^{9}$ The $k-\epsilon$ turbulence model of Launder and Spalding ${ }^{25}$ will soon be incorporated into our solution procedure.

\section{Details of Flux Vector Splitting}

The present finite-difference formulation is based on an upwind scheme because of its superior numerical stability, and efficiency properties compared to those of a centered difference scheme. ${ }^{26}$ The most widely used flux vector splitting methods are those of Steger and Warming, ${ }^{26}$ van Leer, ${ }^{27}$ and Roe. ${ }^{28}$ Recently, there is a considerable interest in extending these methods for the modelling of reactive flows to solve problens emerging from the design of the National Aerospace Plane (NASP), and Air-assisted Orbital Transfer vehicles (AOTV). ${ }^{29,30}$ Details of the generalization of the Steger and Warming flux vector splitting for a perfect gas mixture with variable properties are presented in this section. Since the flux vector $F(Q)$ of $\mathrm{Eq}$. (1) retains its homogenous property for the equation of state considered, the flux vector can be split into two parts,

$$
F=F^{+}+F^{-}
$$

where $F^{+}$is the subvector associated with the non-negative eigenvalues of $A, F^{-}$is the subvector associated with the non-positive eigenvalues of $A$, and $A$ is the Jacobian matrix, $\frac{\partial F}{\partial Q}$. The eigenvalues 
of the matrix, $A$ are given by

$$
\begin{gathered}
\lambda_{1}=\lambda_{2}=\lambda_{3}=\lambda_{6}=\lambda_{7}=U+\xi_{t} \\
\lambda_{4}=U+\xi_{t}+a \Delta \xi \\
\lambda_{5}=U+\xi_{t}-a \Delta \xi
\end{gathered}
$$

where $\Delta \xi=\left(\xi_{x}^{2}+\xi_{y}^{2}+\xi_{z}^{2}\right)^{\frac{1}{2}}, a=\sqrt{\left(\frac{\tilde{C}_{p m}}{\tilde{C}_{v m}}\right) P_{p}^{P}}$ is the speed of sound, $\tilde{C}_{p m}=\sum_{i=1}^{N} y_{i} C_{p i}, \tilde{C}_{u m}=$ $\tilde{C}_{p m}-\tilde{R}$, and $\tilde{R}=R_{u} \sum_{i=1}^{N} \frac{y_{i}}{W_{i}}$. It can also be shown that

$$
F^{+}=M \Lambda^{+} M^{-1} Q, \quad F^{-}=M \Lambda^{-} M^{-1} Q
$$

where the diagonal elements of the matix, $\Lambda^{ \pm}$, are given by

$$
\begin{gathered}
\lambda_{1}^{ \pm}=\lambda_{2}^{ \pm}=\lambda_{3}^{ \pm}=\lambda_{6}^{ \pm}=\lambda_{7}^{ \pm}=\frac{1}{2}\left(U+\xi_{t} \pm\left|U+\xi_{t}\right|\right) \\
\lambda_{4}^{ \pm}=\frac{1}{2}\left(U+\xi_{t}+a \Delta \xi \pm\left|U+\xi_{t}+a \Delta \xi\right|\right) \\
\lambda_{5}^{ \pm}=\frac{1}{2}\left(U+\xi_{t}-a \Delta \xi \pm\left|U+\xi_{t}-a \Delta \xi\right|\right)
\end{gathered}
$$

and

$M=$

$$
\left(\begin{array}{lllllll}
1 & 0 & 0 & \frac{\rho}{\sqrt{2 a}} & \frac{\rho}{\sqrt{2 a}} & 0 & 0 \\
u & \tilde{\xi}_{y} \rho & 0 & \frac{\rho}{\sqrt{2 a}}\left(u+\tilde{\xi}_{z} a\right) & \frac{\rho}{\sqrt{2 a}}\left(u-\tilde{\xi}_{x} a\right) & 0 & 0 \\
v & -\tilde{\xi}_{x} \rho & \tilde{\xi}_{x} \rho & \frac{\rho}{\sqrt{2 a}}\left(v+\tilde{\xi}_{y} a\right) & \frac{\rho}{\sqrt{2 a}}\left(v-\tilde{\xi}_{y} a\right) & 0 & 0 \\
w & 0 & -\tilde{\xi}_{y} \rho & \frac{\rho}{\sqrt{2 a}}\left(w+\tilde{\xi}_{z} a\right) & \frac{\rho}{\sqrt{2 a}}\left(w-\tilde{\xi}_{x} a\right) & 0 & 0 \\
e-\tilde{C}_{v m} T & \rho\left(\tilde{\xi}_{y} u-\tilde{\xi}_{x} v\right) & \rho\left(\tilde{\xi}_{z} v-\tilde{\xi}_{y} w\right) & \frac{\rho}{\sqrt{2 a}}(h+\tilde{U} a) & \frac{\rho}{\sqrt{2 a}}(h-\tilde{U} a) & \rho \tilde{h}_{1} & \rho \tilde{h}_{2} \\
y_{1} & 0 & 0 & \frac{\rho \psi_{u}}{2 a} & \frac{\rho y^{2}}{2 a} & \rho & 0 \\
y_{2} & 0 & 0 & \frac{\rho \psi_{2}}{\sqrt{2 a}} & \frac{\rho y_{2}}{\sqrt{2 a}} & 0 & \rho
\end{array}\right),
$$

$M^{-1}=$ 


$$
\left(\begin{array}{lllllll}
1+\frac{\gamma-1}{a^{2}} \tilde{h}_{0} & \frac{\gamma-1}{a^{2}} u & \frac{\gamma-1}{a^{2}} v & \frac{\gamma-1}{a^{2}} w & -\frac{\gamma-1}{a^{2}} & \frac{\gamma-1}{a^{2}} \tilde{h}_{1} & \frac{\gamma-1}{a^{2}} \tilde{h}_{2} \\
\frac{\left(-u+\bar{\xi}_{x} \tilde{U}\right)}{\rho \tilde{\xi}_{y}} & \frac{\left(1-\bar{\xi}_{z}^{2}\right)}{\tilde{\xi}_{y} \rho} & -\frac{\tilde{\xi}_{z}}{\rho} & -\frac{\tilde{\xi}_{z} \tilde{\xi}_{z}}{\rho \tilde{\xi}_{y}} & 0 & 0 & 0 \\
\frac{\left(w-\bar{\xi}_{z} \tilde{U}\right)}{\rho \tilde{\xi}_{y}} & \frac{\tilde{\xi}_{z} \tilde{\xi}_{z}}{\rho \tilde{\xi}_{y}} & \frac{\bar{\xi}_{z}}{\rho} & -\frac{\left(1-\tilde{\xi}_{z}^{2}\right)}{\tilde{\xi}_{y} \rho} & 0 & 0 & 0 \\
-\frac{1}{\sqrt{2} \rho a}[\tilde{U} a & \frac{1}{\sqrt{2} \rho a}\left[\tilde{\xi}_{x} a\right. & \frac{1}{\sqrt{2} \rho a}\left[\tilde{\xi}_{y} a\right. & \frac{1}{\sqrt{2} \rho a}\left[\tilde{\xi}_{z} a\right. & \frac{\gamma-1}{\sqrt{2} \rho a} & -\frac{\gamma-1}{\sqrt{2} \rho a} \tilde{h}_{1} & -\frac{\gamma-1}{\sqrt{2} \rho a} \tilde{h}_{2} \\
\left.+(\gamma-1) \tilde{h}_{0}\right] & -(\gamma-1) u] & -(\gamma-1) v] & -(\gamma-1) w] & & & \\
-\frac{1}{\sqrt{2} \rho a}[-\tilde{U} a & \frac{1}{\sqrt{2} \rho a}\left[-\tilde{\xi}_{x} a\right. & \frac{1}{\sqrt{2} \rho a}\left[-\tilde{\xi}_{y} a\right. & \frac{1}{\sqrt{2} \rho a}\left[-\tilde{\xi}_{z} a\right. & \frac{\gamma-1}{\sqrt{2} \rho a} & -\frac{\gamma-1}{\sqrt{2} \rho a} \tilde{h}_{1} & -\frac{\gamma-1}{\sqrt{2} \rho a} \tilde{h}_{2} \\
\left.+(\gamma-1) \tilde{h}_{0}\right] & -(\gamma-1) u] & -(\gamma-1) v] & -(\gamma-1) w] & & & \\
-\frac{u}{\rho} & 0 & 0 & 0 & 0 & \frac{1}{\rho} & 0 \\
-\frac{\mu_{2}}{\rho} & 0 & 0 & 0 & 0 & 0 & \frac{1}{\rho}
\end{array}\right)
$$

and

$$
\begin{aligned}
& \tilde{h}_{0}=\bar{h}_{0}+\int_{T_{r e f}}^{T} \bar{C}_{p s} d T-\frac{\tilde{R}}{R_{u}} \bar{W}_{0} \tilde{C}_{p m} T-\frac{1}{2}\left(u^{2}+v^{2}+w^{2}\right) \\
& \tilde{h}_{1}=\bar{h}_{1}+\int_{T_{\text {ref }}}^{T} \bar{C}_{p 1} d T-\frac{\tilde{R}}{R_{u}} \bar{W}_{1} \tilde{C}_{p m} T \\
& \tilde{h}_{2}=\bar{h}_{2}+\int_{T_{\text {ref }}}^{T} \bar{C}_{p 2} d T-\frac{\tilde{R}}{R_{u}} \bar{W}_{0} \tilde{C}_{p m} T
\end{aligned}
$$

and also $\gamma=\tilde{C}_{p m} / \tilde{C}_{v m}, \tilde{\xi}_{x}=\frac{\xi_{z}}{\Delta \xi}, \tilde{\xi}_{v}=\frac{\xi_{y}}{\Delta \xi}, \tilde{\xi}_{x}=\frac{\xi_{x}}{\Delta \xi}$, and $\tilde{U}=\tilde{\xi}_{x} u+\tilde{\xi}_{y} v+\tilde{\xi}_{x} w$.

The resulting components of the split fluxes $F^{ \pm}$are given by 


$$
\begin{aligned}
& F_{1}^{ \pm}=\frac{\bar{C}_{\mathrm{em}}}{\tilde{C}_{p m}} \rho\left[\lambda_{1}^{ \pm} \frac{\bar{R}}{\tilde{C}_{v m}}+\frac{\lambda_{\mathrm{t}}^{ \pm}}{2}+\frac{\lambda_{s}^{ \pm}}{2}\right] \\
& F_{2}^{ \pm}=\frac{\bar{C}_{v m}}{\tilde{C}_{p m}} \rho D\left[\lambda_{1}^{ \pm} \frac{\tilde{R}}{\tilde{C}_{v m}} u+\frac{\lambda_{\bar{f}}^{ \pm}}{2}\left(u+\tilde{\xi}_{x} a\right)+\frac{\lambda_{\xi}^{ \pm}}{2}\left(u-\tilde{\xi}_{x} a\right)\right] \\
& F_{3}^{ \pm}=\frac{\bar{C}_{v m} \rho D}{\tilde{C}_{p m}} \rho\left[\lambda_{1}^{ \pm} \frac{\bar{R}}{\bar{C}_{v m}} v+\frac{\lambda_{t}^{ \pm}}{2}\left(v+\tilde{\xi}_{y} a\right)+\frac{\lambda_{s}^{ \pm}}{2}\left(v-\tilde{\xi}_{y} a\right)\right] \\
& F_{\ddagger}^{ \pm}=\frac{\tilde{C}_{\mathrm{em}} \rho}{\tilde{C}_{p m}} \rho\left[\lambda_{1}^{ \pm} \frac{\tilde{R}}{\tilde{C}_{v m}} w+\frac{\lambda_{t}^{ \pm}}{2}\left(w+\tilde{\xi}_{x} a\right)+\frac{\lambda_{\xi}^{ \pm}}{2}\left(w-\tilde{\xi}_{x} a\right)\right] \\
& F_{\overline{5}}^{ \pm}=\frac{\bar{C}_{u m}}{\tilde{C}_{p m}} \rho D\left[\lambda_{1}^{ \pm} \frac{\tilde{R}}{\tilde{C}_{v m}}\left(h-\tilde{C}_{p m} T\right)+\frac{\lambda_{t}^{ \pm}}{2}(h+\tilde{U} a)+\frac{\lambda_{5}^{ \pm}}{2}(h-\tilde{U} a)\right] \\
& F_{6}^{ \pm}=y_{1} F_{1}^{ \pm} \\
& F_{7}^{ \pm}=y_{2} F_{1}^{ \pm}
\end{aligned}
$$

In this section, we have presented the derivation of the the split fluxes associated with the flux vector $F(Q)$. The corresponding split fluxes associated with the vectors $G(Q)$ and $H(Q)$ can be derived in a similar way.

\section{Details of the Numerical Method}

Solution for the gas-phase equations is obtained by making use of a finite-volume, LowerUpper (LU) decomposition scheme. The governing equations are linearized in a delta form where the nonlinear terms associated with finite-rate chemistry and convection are treated implicitly, while the diffusion terms and the source terms arising through liquid-phase interaction are treated explicitly. The time-linearized governing equations in delta form are

$$
\begin{gathered}
{\left[D^{n+1} I+\Delta t\left(\delta_{\xi}^{-} A^{+}+\delta_{\eta}^{-} B^{+}\right.\right.} \\
\left.\left.+\delta_{\zeta}^{-} C^{+}+\delta_{\xi}^{+} A^{-}+\delta_{\eta}^{+} B^{-}+\delta_{\xi}^{+} C^{-}-L\right)\right] \Delta \hat{Q} \\
=-\hat{Q}^{n} \Delta D+\Delta t \vec{r}
\end{gathered}
$$

where $A^{ \pm}=\frac{\partial \vec{F}^{ \pm}}{\partial \hat{Q}}, B^{ \pm}=\frac{\partial \vec{G}^{ \pm}}{\partial \hat{Q}}, C^{ \pm}=\frac{\partial \vec{H}^{ \pm}}{\partial \hat{Q}}, L=\frac{\partial \vec{S}_{f}}{\partial \hat{Q}}, \Delta t$ is the time step size, $\delta^{+}$and $\delta^{-}$are forward and backward differences, respectively, and

$$
\begin{gathered}
\Delta \vec{Q}=\hat{Q}^{n} \Delta D+D^{n+1} \Delta \hat{Q}, \\
\vec{r}=-\delta_{\xi}^{-} \vec{F}^{+}-\delta_{\eta}^{-} \vec{G}^{+}-\delta_{s}^{-} \vec{H}^{+}-\delta_{\xi}^{+} \vec{F}^{-}-\delta_{\eta}^{+} \vec{G}^{-} \\
-\delta_{\xi}^{+} \vec{H}^{-}+\delta_{\xi} \vec{F}_{v}+\delta_{\eta} \vec{G}_{v}+\delta_{\zeta} \vec{H}_{v}+\vec{S}_{c}+\vec{S}_{l}
\end{gathered}
$$


Upon factoring Eq. (20) we obtain the following sequence:

$$
\begin{gathered}
{\left[D^{n+1} I+\Delta t\left(\delta_{\xi}^{+} A^{-}+\delta_{\eta}^{+} B^{-}+\delta_{\xi}^{+} C^{-}-L\right)\right] \Delta \hat{Q}^{*}=-\hat{Q}^{n} \Delta D+\Delta t \vec{r}} \\
{\left[D^{n+1} I+\Delta t\left(\delta_{\xi}^{-} A^{+}+\delta_{\eta}^{-} B^{+}+\delta_{\zeta}^{-} C^{+}\right)\right] \Delta \hat{Q}=I D^{n+1} \Delta \hat{Q}^{*}}
\end{gathered}
$$

It is noteworthy that to be consistent with the objective of deriving a finite-volume code, the split-flux differences in Eq. (21) are implemented according to Monotone Upstream-Centered Schemes for Conservation Laws (MUSCL)-type differencing. ${ }^{31,32}$ The fluxes at the cell faces are first obtained by a fully upwind first-order accurate interpolation, and then centered differences are used for both the forward and backward spatial operators evaluated at the cell centers. Centered differences are also used for evaluating the spatial operators associated with the viscous terms.

For the dynamic grid calculations, the metric quantities are evaluated at time level $n+1$, and $D^{n+1}$ is evaluated from the solution of Eq. (9) by using an explicit method. The numerical grid is generated by an algebraic technique ${ }^{8}$ with the help of the grid-generation code taken from the LEWIS-3D code. ${ }^{9}$

By adopting an algorithm taken from the RPLUS-3D code, ${ }^{12}$ the present code is vectorized rather efficiently by operating on all points in a diagonal plane of the computational space, simultaneously. The diagonal plane is one on which $i+j+k=$ constant. The integration proceeds during the backward and forward substitution steps from one corner node of the computational grid and ends at a corner node which is farthest from the initial corner node.

The boundary conditions are implemented explicitly by defining a layer of phantom cells outside the boundaries of the computational domain. It is also noteworthy that the left-hand side operators of Eqs. (23) to (24) require block diagonal inversions.

\section{Results and Discussion}

Here we present the results of our preliminary computations for a single case corresponding to the operating conditions listed in Table 1 , where subscripts $0, c, r$, and $h$ represent opening, closing, rotor, and housing, respectively, $V_{d, i}$ is the initial droplet velocity, and $T_{d, i}$ is the initial droplet temperature. Isothermal wall conditions are implemented in the case considered. 


\begin{tabular}{|l|l|}
\hline \multicolumn{2}{|c|}{ Table 1. Operating Conditions } \\
\hline $\begin{array}{l}\text { Engine Parameters } \\
\text { (see Fig. 1) }\end{array}$ & $\begin{array}{l}\text { Generating Radius(R) }=0.1064 \mathrm{~m} \\
\text { Eccentricity }(\mathrm{E})=0.01542 \mathrm{~m} \\
\text { Clearance(C) }=0.004 \mathrm{~m} \\
\text { Chamber Width }(\mathrm{W})=0.07 \mathrm{~m} \\
\text { Port Width }\left(W_{P}\right)=0.05 \mathrm{~m}\end{array}$ \\
\hline Engine Speed & $4000 \mathrm{rpm}$ \\
\hline Intake Port & $\begin{array}{l}\theta_{O}=-1.26 \mathrm{rad}, \theta_{C}=5.96 \mathrm{rad}, P_{\text {int }}=1.25 \mathrm{~atm}, \\
T_{\text {int }}=300 \mathrm{~K}, Y_{\text {l,int }}=0\end{array}$ \\
\hline Exhaust Port & $\theta_{O}=-5.96 \mathrm{rad}, \theta_{C}=1.07 \mathrm{rad}, P_{\text {ezh }}=0.85 \mathrm{~atm}$ \\
\hline Fuel Injector & $\begin{array}{l}\theta_{O}=8.3 \mathrm{rad}, \theta_{C}=8.75 \mathrm{rad}, V_{d, i}=100 \mathrm{~m} / \mathrm{s}, \\
T_{d, i}=300 \mathrm{~K}\end{array}$ \\
\hline Spark ignition Timings & $\theta_{O}=8.35 \mathrm{rad}, \theta_{C}=8.475 \mathrm{rad}$ \\
\hline $\begin{array}{l}\text { Temperature of Rotor } \\
\text { and Housing Surfaces }\end{array}$ & $T_{h}=T_{r}=400 \mathrm{~K}$ \\
\hline
\end{tabular}

The computations are performed with a variable time-step corresponding to a maximum CFL number of 20 , and on a grid with a mesh size of $i=31, j=16$, and $k=20$, where $i, j$, and $k$ represent the coordinate surfaces in the direction extending from the trailing-edge surface to the leading-edge surface of the combustor, from the rotor to housing surface, and from the side wall to the symmetry plane of the domain between the end-to-end side walls, respectively.

As described in Section II, the computations are initiated before the opening of the exhaust port, and are terminated after the completion of combustion process. Figure 2 shows the variation of the engine volume versus crank angle. The indicated engine volume is obtained from the numerical integration of the individual computational-cell volumes. The figure demonstrates the ability of the numerical method to accurately reproduce the combustor volume changes corresponding to a maximum and minimum displacement volume of 750 c.c. and 115 c.c, respectively, yielding a compression ratio of about 6.5 .

Figure 3 shows flow patterns (particle traces) during the middle of the exhaust (Fig. 3a), of the intake (Fig. 3b), and of the simultaneous opening of both exhaust and intake ports (Fig. 3c) together with the schematic of a Wankel engine (Fig. 3d). The particle traces are coloured based upon the local value of the internal energy e. Fig. 3a shows clearly that the bulk fluid motion of the residual gas is essentially directed out of the engine chamber through the exhaust port. The fluid motion is clearly seen to be influenced by the combined effect of the positive pressure difference that exists between the chamber and back pressure, and of the rotor motion on the fluid near the rotor surface. Figure $3 \mathrm{c}$ shows that while part of the residual gas is escaping through the exhaust port, fresh air is entering the chamber through the intake port. Because of the difference that exists between the intake and exhaust pressure (1.25 and $0.85 \mathrm{~atm})$, part of the fresh air emerging from the intake is drawn towards the exhaust before it recirculates in the rotor pocket. 
Figure $3 b$ shows a complex flow pattern created by a strong jet of fresh air emerging from the intake in a cross flow. The cross flow is created by the clockwise movement of the rotor surface. The flow pattern reveals the existence of two clearly defined recirculation (low-pressure) regions on both sides of the intake along the ith direction. Upon the impingement of the intake jet on the rotor surface, the jet, near the leading edge, is partly drawn into the low-pressure region, and part of the fluid is carried over backwards through the openings between the outer edge of the jet and the side-walls.

Figure 4, which is similar to Fig. 3b, shows an experimental flow-visualization result obtained by Hamady et al. ${ }^{33}$ using a transparent-sided rotary engine motoring test rig. "Microballoon" seeding material was illuminated by a pulsed laser light and recorded by high-speed photography. At the leading edge of the jet, a recirculatory flow pattern quite similar to that shown in Fig. $3 \mathrm{~b}$ is clearly seen. Although quantitative comparison has not yet been attempted, the degree of qualitative agreement noted here is quite encouraging. The trailing vortex, which is clearly shown in Fig. 3b, is beyond the field of view of Fig. 4.

The droplet trajectories at $\theta=8.5$ and $8.75 \mathrm{rad}$ are shown in Figs. 5 and 6 . The polydisperse character of the spray is represented by different sized circles which are indicative of the size of the initial droplets. The initial droplet sizes range between $10 \mu m \leq r_{k, i} \leq 30 \mu m$, and the initial droplet Reynolds numbers vary between $75 \leq R e_{k, i} \leq 600$. The wide disparity in $R e_{k, i}$ is a result of the steep rise in the chamber pressure due to combustion from 6 to 35 atm during the time of fuel injection. Because of the large initial momentum associated with these particles, they retain their initial path as described by their initial conditions. It takes about $1.5 \mathrm{~ms}$ for the largest particles to vaporize. The deflection of the particles in the direction of the gaseous flow is evident from Fig. 6 as the droplets become smaller due to evaporation.

The temperature distribution within the combustion chamber during the early stages of flame propagation at $\theta=8.5 \mathrm{rad}$, and after combustion at $\theta=10 \mathrm{rad}$ is shown in Figs. 7 and 8 , respectively. In Fig. 7 , the highest temperature region $(2900 \mathrm{~K})$ is confined to the region near the rotor pocket, where the liquid fuel is injected. The temperatures are lower near the walls and in the clearance regions near the leading and trailing apex seals, where the heat transfer to the walls is greatest because of the high surface-to-volume ratio. In Fig. 8, the highest temperature region extends all the way from the region near the rotor pocket to the region near the leading apex seal, while the temperatures are lower in the region near the trailing apex seal. During the expansion stroke, the region near the leading apex seal becomes wider, causing a decrease in the heat transfer rate to the walls in that region, while heat transfer to that region within the chamber interior increases due to convection of the fluid as influenced by the rotor rotation. An opposite trend is observed in the region near the trailing seal.

The gaseous-fuel mass fraction contours at $\theta=8.5$ and 8.75 rad are presented in Figs. 9 and 10. It is noteworthy that in presenting some of the results involving the iso-contour lines of fuel concentration in Figs. 9 and 10, and also of pressure in Fig. 13, some of the contour lines representing the near-maximum values are shown in dotted lines. Thus, Figs. 9-10 show that the 
region near the fuel injector location is fuel rich. Diffusion of the fuel concentration with time and the influence of convection on the distribution of the fuel concentration more towards the leading region is also evident from the comparison of these figures. While the stratified charge gives rise to a diffusion flame, a careful examination also reveals that part of the evaporated-fuel and oxidizer mixure burns like a premixed flame. This is demonstrated by an absence of fuel concentration in the region near the rotor pocket surface where the fuel concentration results otherwise from the presence of liquid fuel in that region, as shown in Figs. 6 and 7. It is more likely that the combustion characteristics in that region might be influenced by an isolated-combusting droplet behaviour.

Fig. 11 shows the angular variation of the amount of the evaporated-fuel and also the amount of reacted-fuel. The results are obtained by integrating the source term contributions of the gas-phase equations arising from the production of fuel due to evaporation and also from the consumption of reactants due to combustion. It is noteworthy that the total amount of liquid fuel injected is determined based on an equivalence ratio of 0.7 . The results show that the total time for complete vaporization and also combustion is less than $2 \mathrm{~ms}$. The slope of these curves indicates that most of the fuel, after it evaporates, reacts quickly with oxidizer to form products. This in turn implies that most of the fuel burns in a premixed-flame environment. Since the vaporization rate and ignitiondelay characteristics of this model were not known in advance, however, the fuel injection and spark timings were arbitrarily chosen to be $66^{\circ}$ and $63^{\circ}$, respectively, before TDC. These conditions, which in retrospect are clearly non-optimal, correspond to very advanced fuel injection and spark timings. Under more optimal engine operating conditions, the fuel injection evidently sho: $d$ not begin before $30^{\circ}$ to $45^{\circ}$ from the TDC. Both the vaporization and combustion characteristics might be quite different if these timings are chosen according to optimized operating conditions. Future work will address the optimization of these timings in terms of the overall combustion behaviour. Note also that because of these advanced timings, additional energy has to be supplied for the work to be performed during the remainder of the compression process from the time after combustion to the time before the TDC is reached.

Figure 12 shows the velocity vector plots at four different crank angles: Fig. 12a at the beginning of fuel injection, Figs. $12 \mathrm{~b}$ and $12 \mathrm{c}$ during combustion, and Fig. 12d after combustion. In these velocity vector plots, only three different sizes of arrow symbols are used to distinguish the variation between the maximum and minimum values in magnitude. These plots indicate that the direction of fluid motion near the symmetry plane is mainly determined by the rotor motion; however, for a brief period during the early stages of flame propagation as shown in Fig. 12b, the expanding gases do create a motion in a direction opposite to the main bulk flow. The non-uniformity in pressure between the leading and trailing regions as shown by the pressure contours in Fig. 13 better explains the reason for the strong bulk fluid motion created by the rotor movement. Recall that, as in Figs. 9 and 10, some of the near-maximum contours are shown in dotted lines. These figures do show a more or less gradual decrease of pressure from the trailing to the leading apex seals, and the pressure decreases in the direction of the fluid motion. The higher pressure drop, as 
expected from the substantial friction losses in the regions closer to the apex seals, is also evident from these figures.

\section{Concluding Remarks}

We have presented a description of a new computer code developed for the modelling of stratified-charge rotary engine performance based on the solution of the unsteady, three-dimensional Navier-Stokes equations, with the use of convenient submodels for turbulence, combustion, and sprays. The details of the rotary engine flowfield during exhaust and/or intake processes and compression stroke, and also the details of the mixing, vaporization processes during and after combustion have been presented for a single case with advanced fuel injection and spark ignition timings. The salient features of this work are summarized below:

1. The code takes approximately 3 CPU-hours, when the calculations are performed on a grid with a mesh size of $31 \times 16 \times 20$ on a CRAY Y-MP, for a non-reacting case, and it takes about 7.5 to 10 CPU-hours for a reactive case with sprays. For the non-reactive case, the solution can be marched in time non-iteratively, but, for a reactive case, the solution is obtained by an iterative procedure.

2. One apparent finding of our study is that vaporization appears to be more rate-controlling than mixing during the combustion process, at least in the case that we have studied with advanced fuel injection and spark ignition.

3. There is a good degree of qualitative agreement between the prediction and an experimental flow-visualization pattern of Hamady et al.$^{33}$ obtained during the intake process.

4. The present solution procedure makes use of an extremely simplified constant diffusivity turbulence model. The $k-\epsilon$ turbulence model of Launder and Spalding ${ }^{25}$ will soon be incorporated into our solution procedure.

5. The present combustion model is based on laminar kinetics. While recognizing the fact that no realistic model would be available in the foreseeable future for a proper treatment of the effect of turbulence on combustion reaction rates, the eddy break-up model of Spalding ${ }^{24}$ will be incorporated into our solution procedure in order to account for some effect of turbulence on the combustion processes.

6. After implementing the above-mentioned modifications we will conduct a parametric study in order to optimize the location and also timing of the fuel injector and spark igniter. 


\section{Acknowledgements}

The work of the first author (MSR) was supported under contract NAS3-26266 from the NASA Lewis Research Center. The authors would like to thank Dr. J.-S. Shuen and Mr. J.M. Barton for some helpful discussions held during the course of this work.

\section{References}

1. Danieli, G.A., Keck, J.C., and Heywood, J.B., "Experimental and Theoretical Analysis of Wankel Engine Performance," SAE Paper 780416, 1978.

2. Roberts, J.A., Norman, T.J., Ekchian, J.A., and Heywood, J.B., "Computer Models for Evaluating Premixed and DISC Wankel Engine Performance," SAE Paper 860613, 1986.

3. Bracco, F.V. and Sirignano, W.A., "Theoretical Analysis of Wankel Engine Combustion," Combustion Science and Technology, Vol. 7, No. 3, 1973, pp. 109-123.

4. Grasso, F., Wey, M.-J., Bracco, F.V., and Abraham, J., "Three-Dimensional Computations of Flows in a Stratified-Charge Rotary Engine," SAE Paper 870409, 1987.

5. Abraham, J., Wey, M.-J., and Bracco, F.V., "Pressure Non-Uniformity and Mixing Characteristics in a Stratified Charge Rotary Engine," SAE Paper 880604, 1988.

6. Abraham, J. and Bracco, F.V., "Comparisons of Computed and Measured Pressure in a Premixed-Charge Natural-Gas-Fueled Rotary Engine," Rotary Engine Design: Analysis and Developments, SAE SP-768, SAE, Warrendale, PA, 1989, pp. 117-131.

7. Amsden, A.A., Ramshaw, J.D., O'Rourke, P.J., and Dukowicz, J.K., "KIVA: A Computer Program for Two- and Three- Dimensional Fluid Flows with Chemical Reactions and Fuel Sprays," LA-10245-MS, Los Alamos National Laboratory, Feb. 1985.

8. Shih, T. I.-P., Yang, S.-L., and Schock, H.J., "A Two-Dimensional Numerical Study of the Flow Inside the Combustion Chamber of a Motored Rotary Engine," SAE Paper 860615, 1986.

9. Steinthorsson, E., Shih, T.I-P, Schock, H.J., and Stegman, J., "Calculations of the Unsteady, Three-Dimensional Flow Field Inside a Motored Wankel Engine," SAE Paper 880625, 1988.

10. Li, T., Steinthorsson, E., Shih, T. I.-P., and Nguyen, H.L., "Modeling \& Simulation of Wankel Engine Flow Fields," SAE Paper 900029, 1990.

11. Yamamoto, K.,Rotary Engine, Tokyo Kogyo Co., Ltd., Tokyo, Japan, 1969. 
12. Yu, S.-T., Tsai, Y.-L. P., and Shuen, J.-S., "Three-Dimensional Calculation of Supersonic Reacting Flows Using an LU Scheme," AIAA Paper 89-0391, 1989.

13. Thomas, P.D. and Lombard, C.K., "Geometric Conservation Law and its Application to Flow Computations on Moving Grids," AIAA Journal, Vol. 17, No. 10, Oct. 1978, pp. 1030-1037.

14. Raju, M.S. and Sirignano, W.A., "Spray Computations in a Centerbody Combustor," The 1987 ASME-JSME Thermal Engineering Joint Conference, Vol. 1, P.J. Marto and I. Tanasawa, Eds., ASME, New York, 1987, pp. 61-72.

15. Raju, M.S. and Sirignano, W.A., "Multicomponent Spray Computations in a Modified Centerbody Combustor," AIAA Paper 88-0638, 1988.

16. Kuo, K.K., Priniciples of Combustion, John Wiley \& Sons, Inc., New York, 1986.

17. Faeth,G.M., "Mixing, Transport and Combustion in Sprays," Progress in Energy and Combustion Science, Vol. 13, No. 4, 1987, pp. 293-345.

18. O'Rourke, P.J. and Bracco, F.V., "Modeling of Drop Interactions in Thick Sprays and a Comparison with Experiments," Stratified Charge Automotive Engineering Conference, Institute of Mechanical Engineering, London, England, 1980, Paper C404.

19. Greenberg, J.B. and Tambour, Y., "Far-Field Coalescence Effects in Polydisperse Spray Jet Diffusion Flames," Twenty-First Symposium (International) on Combustion, The Combustion Institute, Pittsburgh, 1986, pp. 655-663.

20. Asheim, J.P., Kirwan, J.E., and Peters, J.E., "Modeling of a Hollow-Cone Liquid Spray Including Droplet Collisions," Journal of Propulsion and Power, Vol.4, No.5, Sept.-Oct. 1988, pp. 391-398.

21. Chiang, C.H., Raju, M.S., and Sirignano, W.A., "Numerical Analysis of Convecting, Vaporizing Fuel Droplet with Variable Properties," AIAA Paper 89-0834, 1989.

22. Haywood, R. and Renksizbulut, M., "On Variable Property, Blowing and Transient Effects in Convective Droplet Evaporation with Internal Circulation," Heat Transfer 1986, Vol. 4, C.L. Tien, V.P. Carey, and J.K. Ferell, Eds., Hemisphere Publishing Corp., New York, 1986, pp.1861-1866.

23. Westbrook, C.K. and Dryer, F.L., "Chemical Kinetic Modelling of Hydrocarbon Combustion," Progress in Energy and Combustion Science, Vol. 10, No. 1, 1984, pp. 1-57.

24. Spalding, D.B., "Mathematical Models of Turbulent Flames: A Review," Combustion Science and Technology, Vol. 13, Nos. 1-6, 1976, pp. 3-25.

25. Launder, B.E. and Spalding, D.B.,Mathematical Models of Turbulence, Academic press, London, 1972. 
26. Steger, J.L. and Warming, R.F., "Flux Vector Splitting of the Inviscid Gasdynamic Equations with Application to Finite-Difference Methods," Journal of Computational Physics, Vol. 40, No. 2, Apr. 1981, pp. 263-293.

27. van Leer, B., "Flux-Vector Splitting for the Euler Equations," 8th International Conference on Numerical Methods in Eluid Mechanics, (Lecture Notes in Physics, Vol. 170), Springer-Verlag, 1982, pp. 507-512.

28. Roe, P.L., "Approximate Riemann Solvers, Parameter Vectors, and Difference Schemes," Journal of Computational Physics, Vol. 43, No. 2, Oct. 1981, pp. 357-372.

29. Liu, Y. and Vinokur, M., "Nonequilibrium Flow Computations. I. An Analysis of Numerical Formulations of Conservation Laws," Journal of Computational Physics, Vol. 83, No. 2, Aug. 1989, pp. 373-397.

30. Shuen, J.-S., Liou, M.-S., and van Leer, B., "Inviscid Flux-Splitting Algorithms for Real Gases with Non-Equilibrium Chemistry," Journal of Computational Physics, in Press, 1989.

31. Belk, D.M., "Unsteady Three-Dimensional Euler Equations Solutions on Dynamic Blocked Grids,” Ph.D. Thesis, Mississippi State University, 1986.

32. Anderson, W.K., "Implicit Multigrid Algorithms for the Three-Dimensional Flux Split Euler Equations," Ph.D. Thesis, Mississippi State University, 1986.

33. Hamady, F., Schock, H., and Stueckon, T., "Airflow Visualization and LDV Measurements in a Motored Rotary Engine Assembly-Part 1: Flow Visualization," SAE Paper $900030,1989$. 

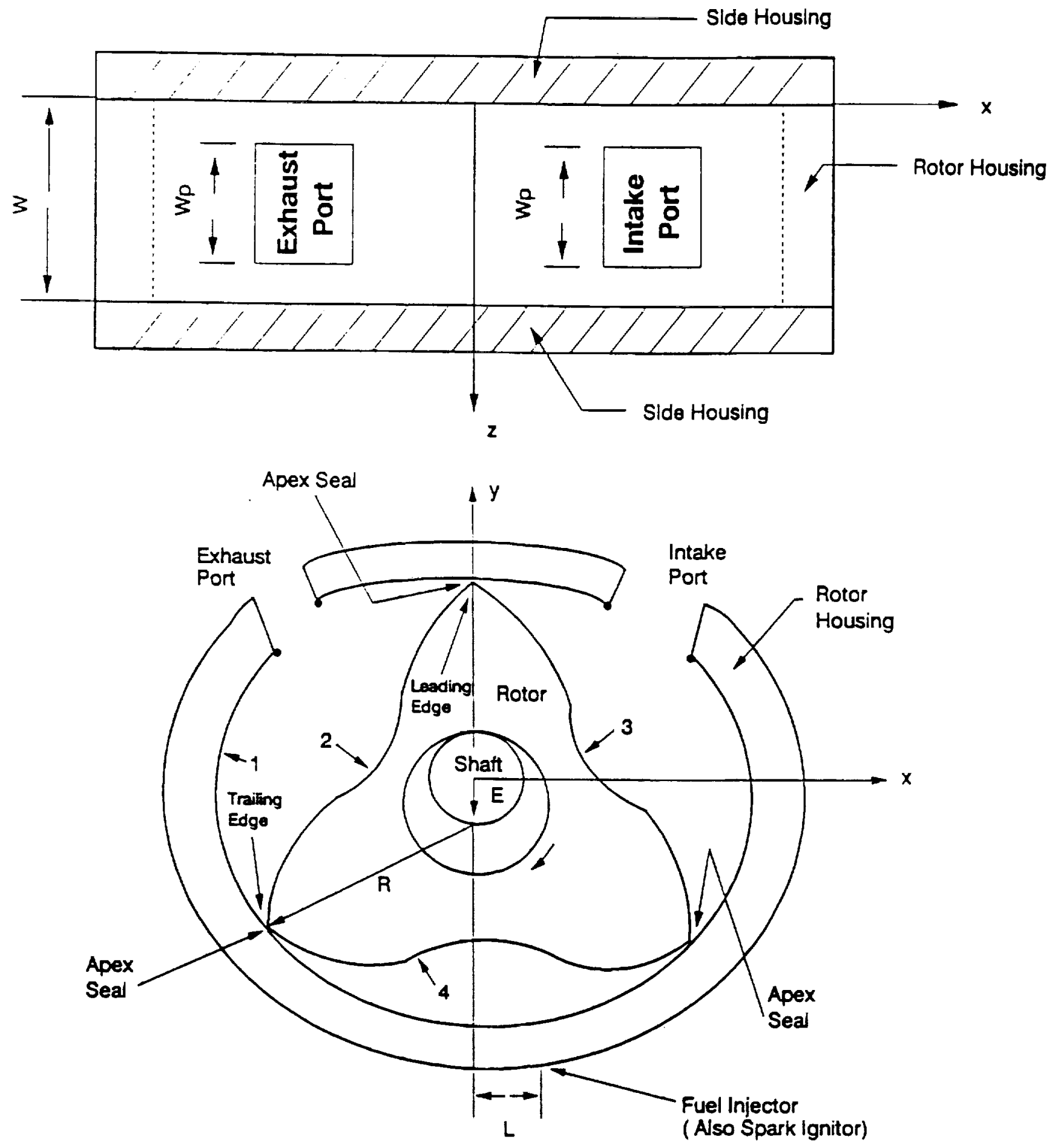

Fig. 1 Schematic of the Wankel engine that was studied. 


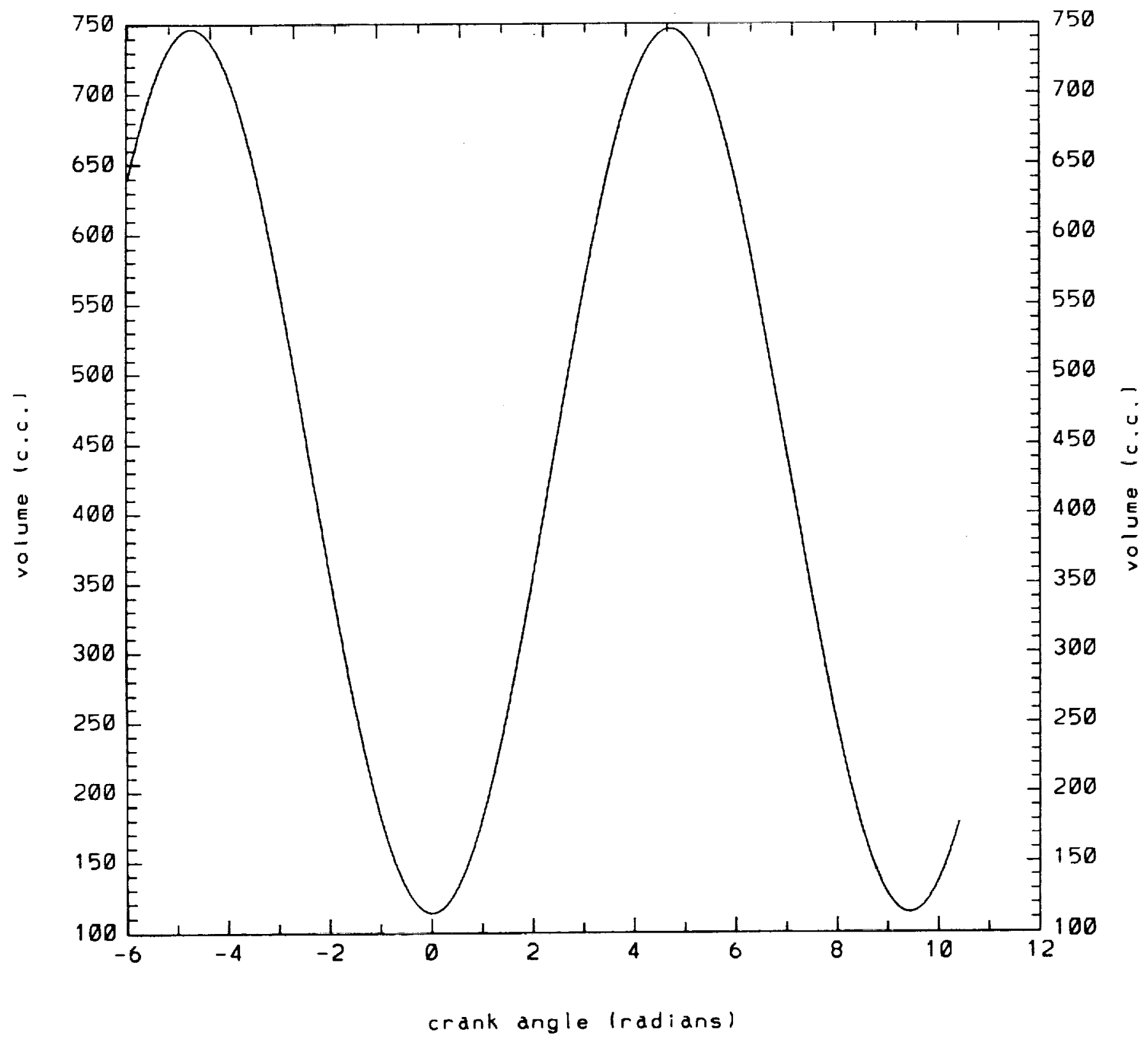

Fig. 2 Variation of the engine volume versus crank angle. 


\section{ORIGHAS PAGE \\ COLOR PHOTOGRAPH}

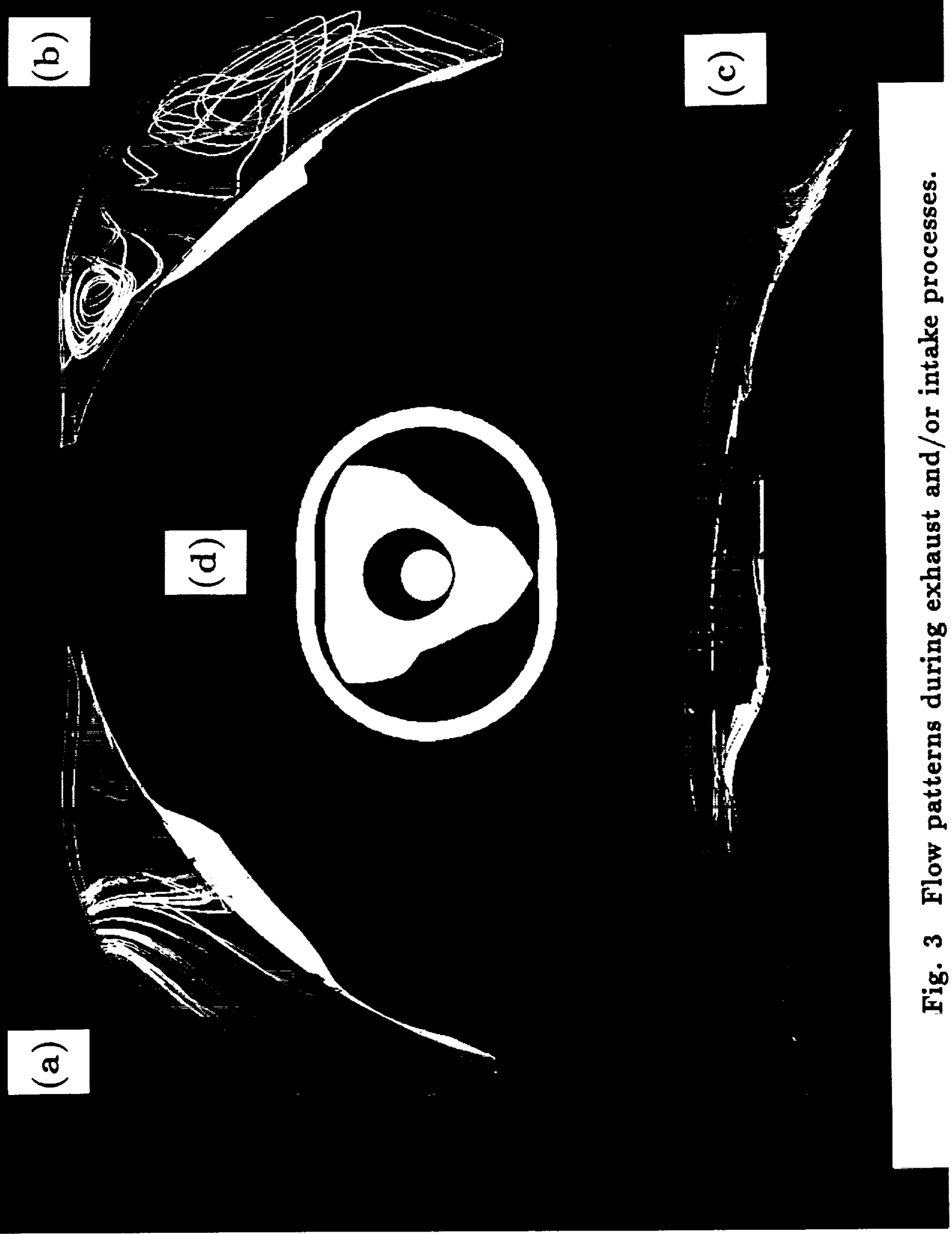




\section{ORIGINAL PAGE}

BLACK AND WHITE PHOTOGRAPH

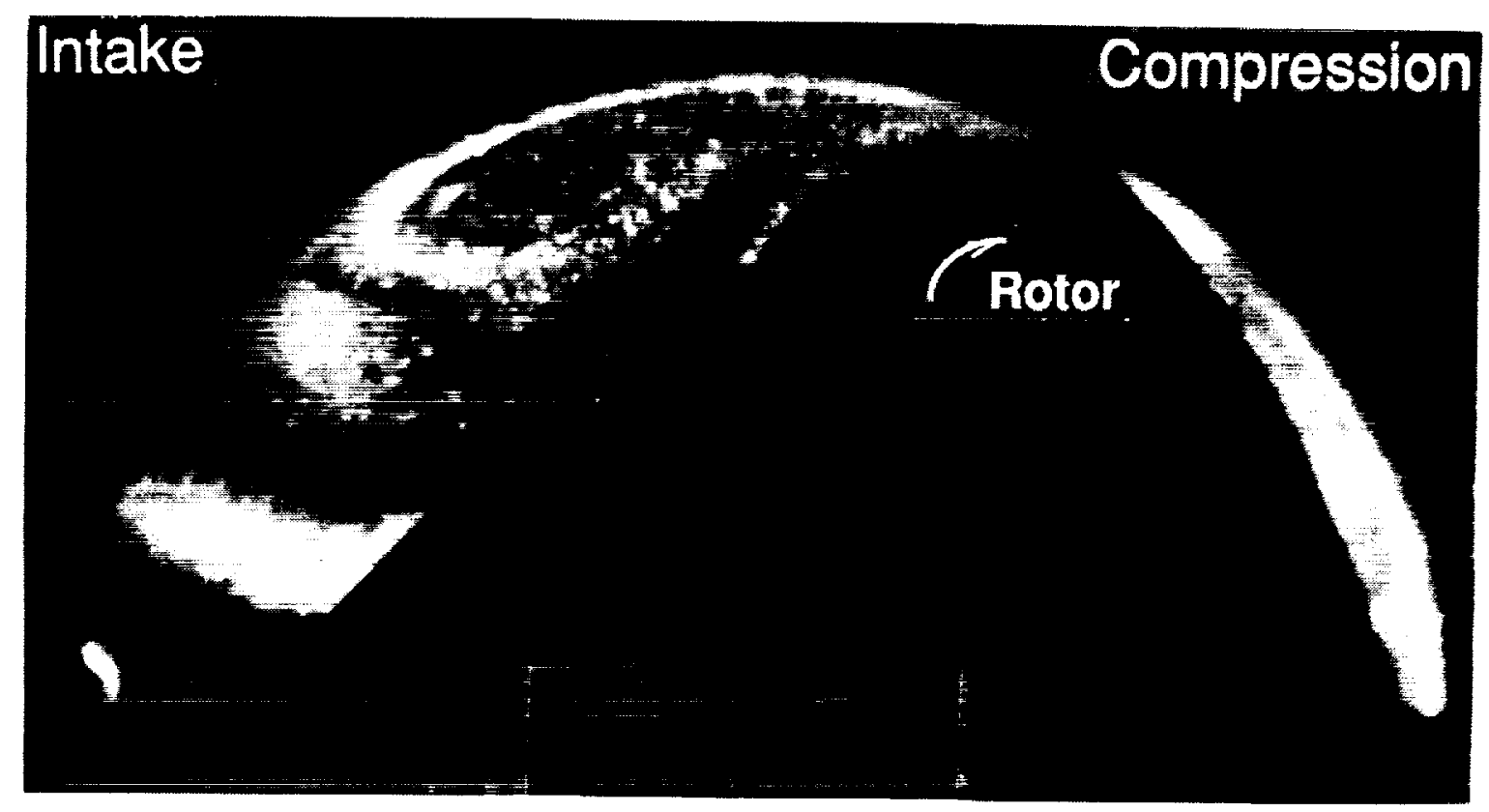

Fig. 4 Experimentally obtained flow pattern during intake at $1170 \mathrm{rpm}$ in a supercharged rotary engine. 

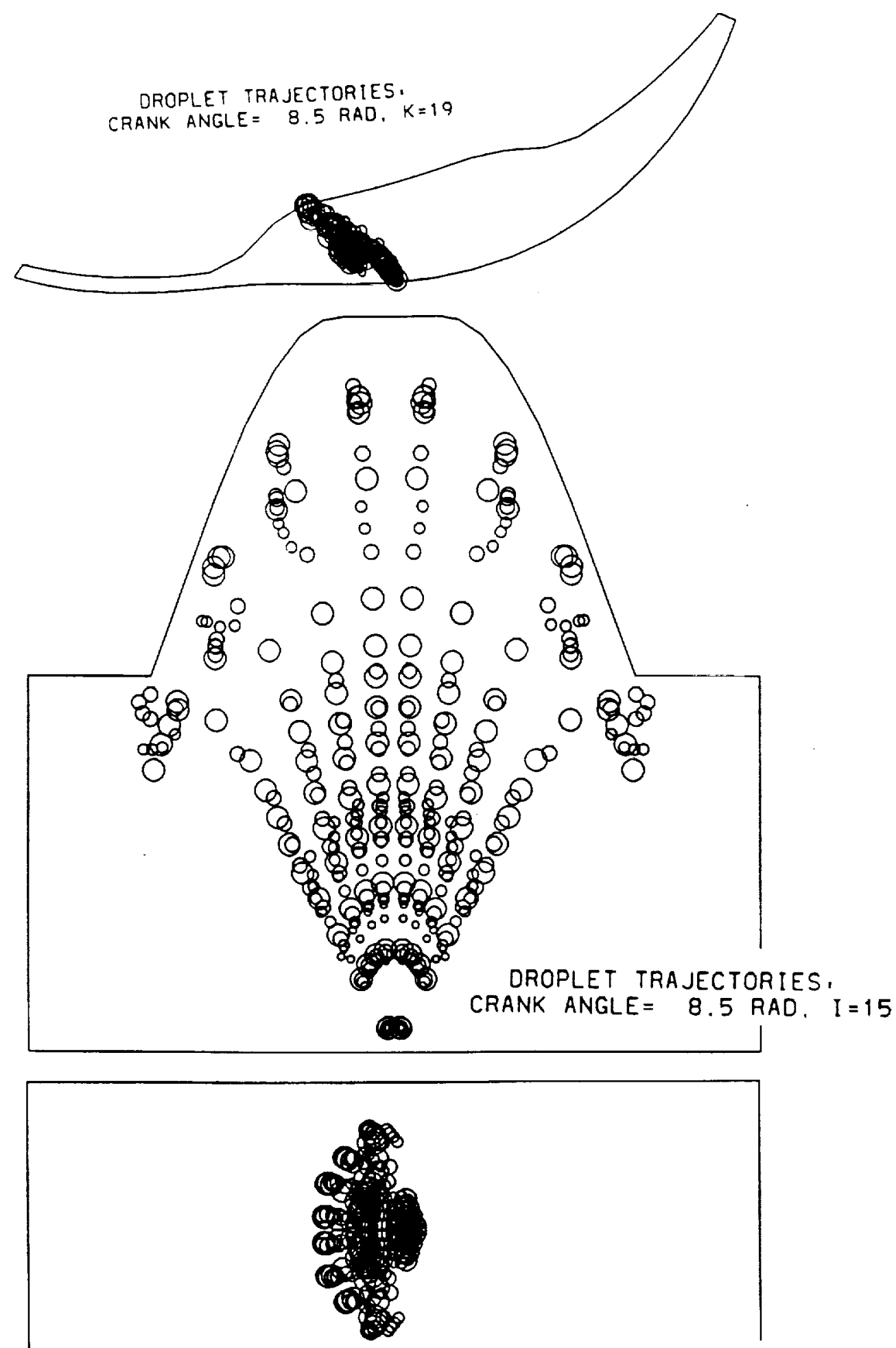

OROPLET TRAJECTORIES, CRANK ANGLE $=8.5$ RAD. $J=1$

Fig. 5 Droplet trajectories at $\theta=8.5 \mathrm{rad}$. 


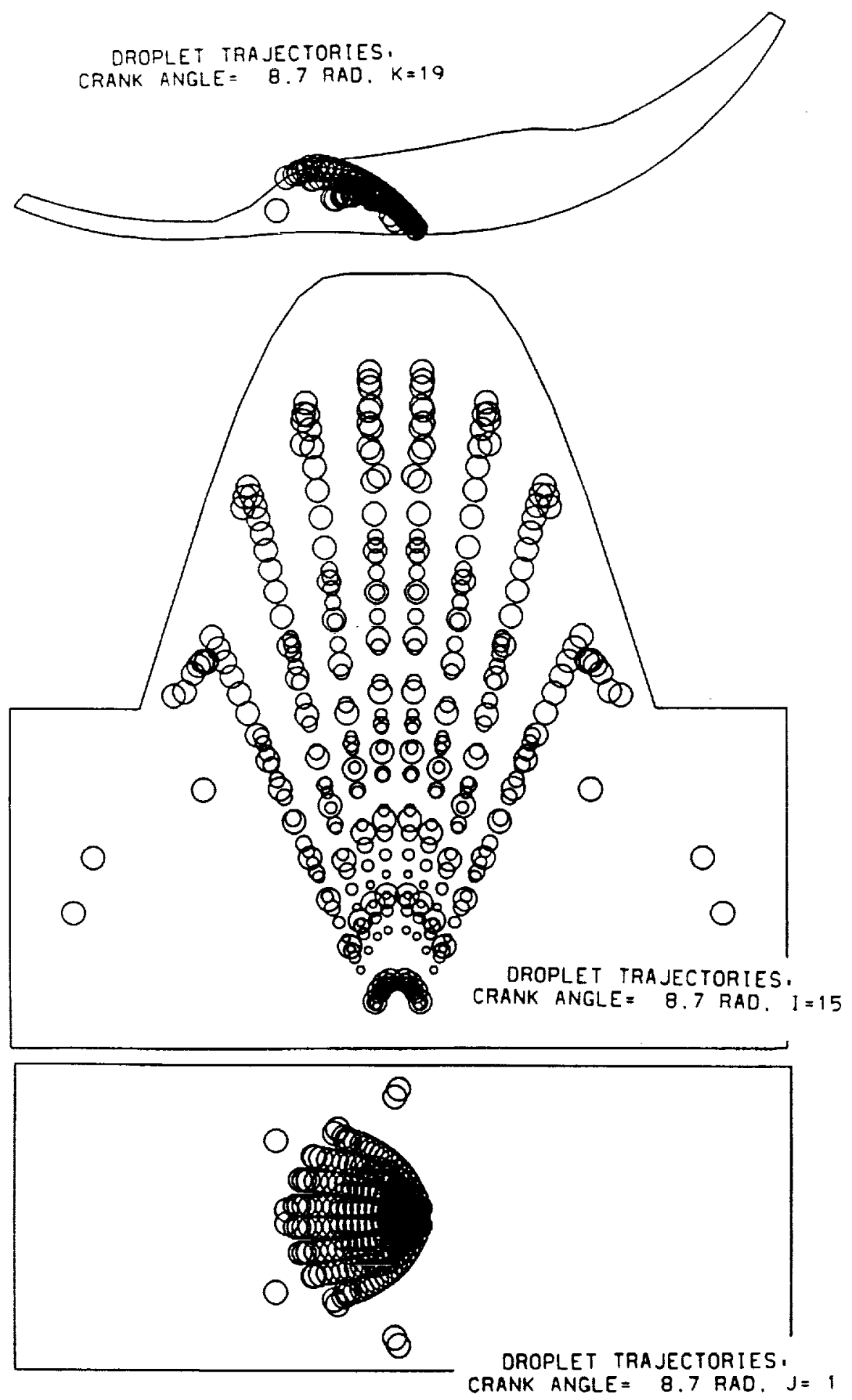

Fig. 6 Droplet trajectories at $\theta=8.75$ rad. 


\section{ORIGINAL PAGE \\ COLOR PHOTOGRAPH}

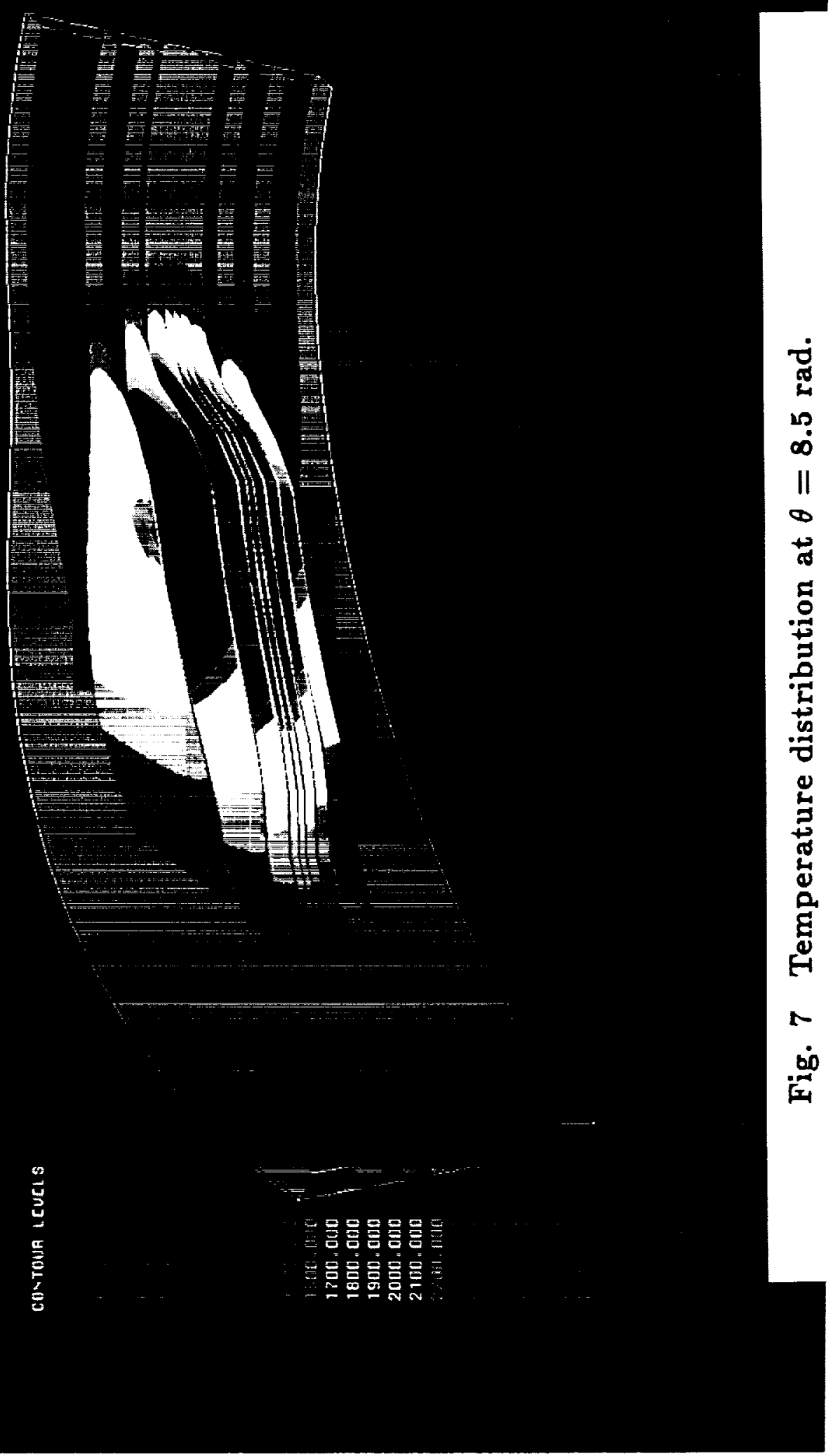





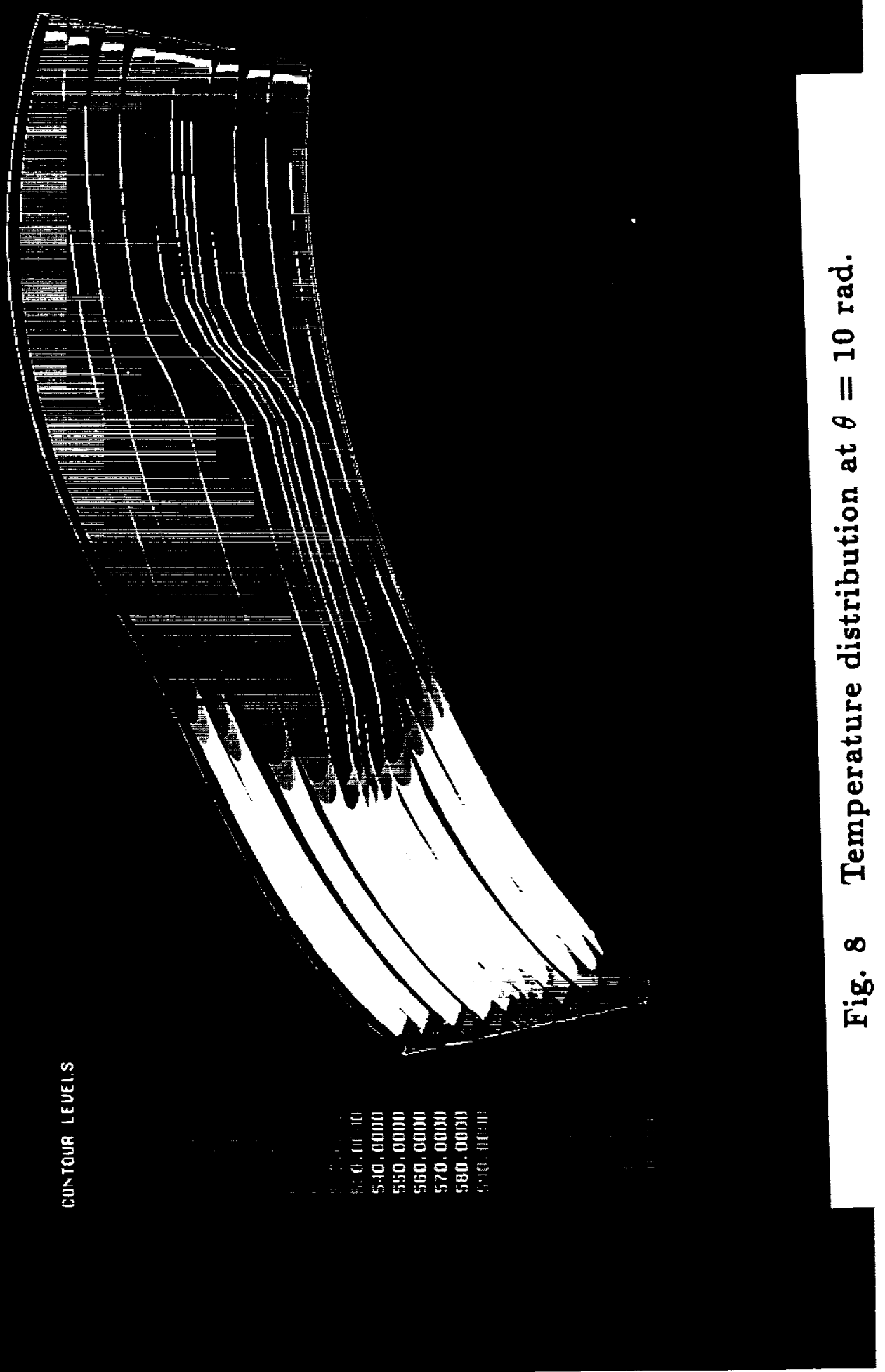





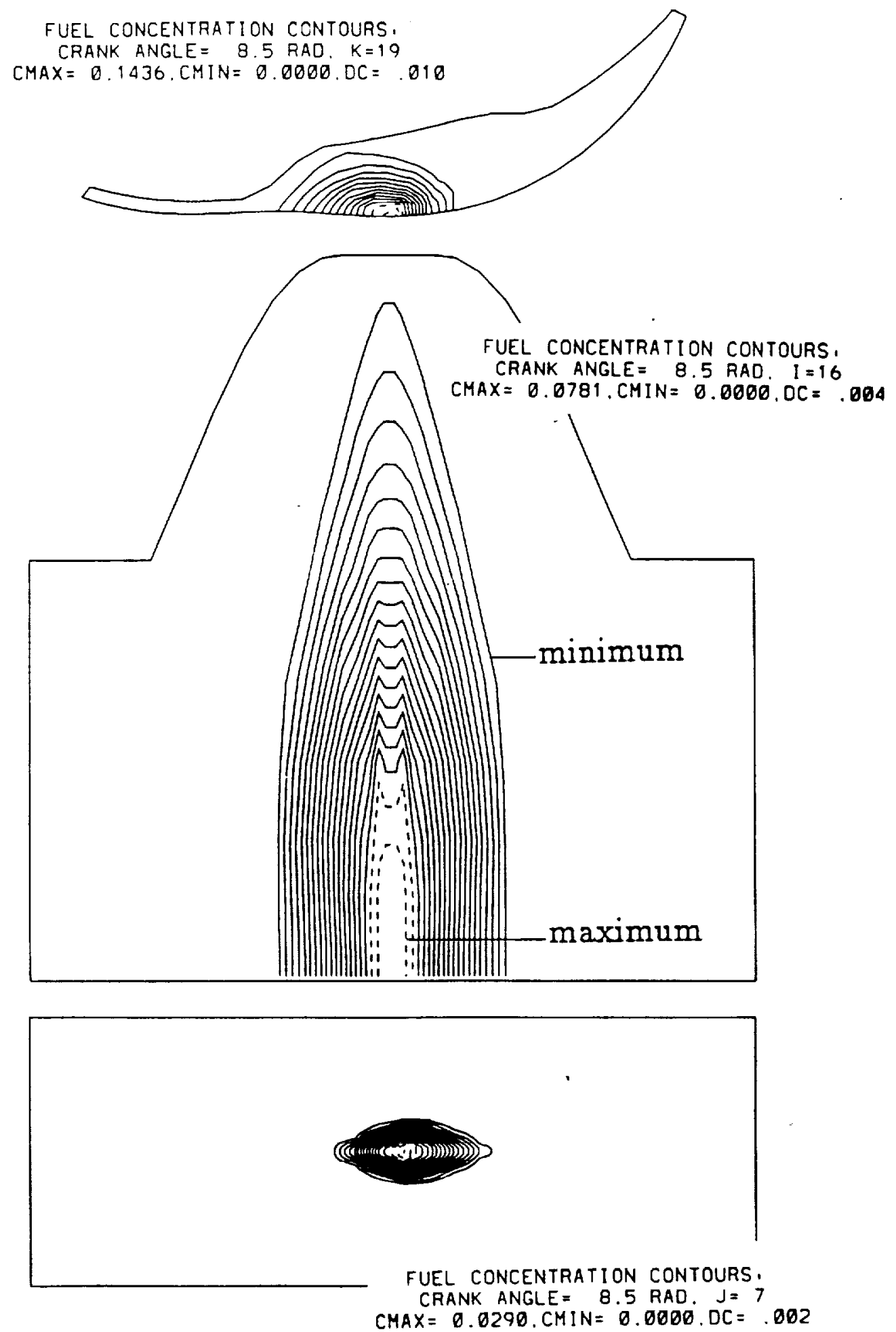

Fig. 9 Fuel mass fraction contours at $\theta=8.5 \mathrm{rad}$. 

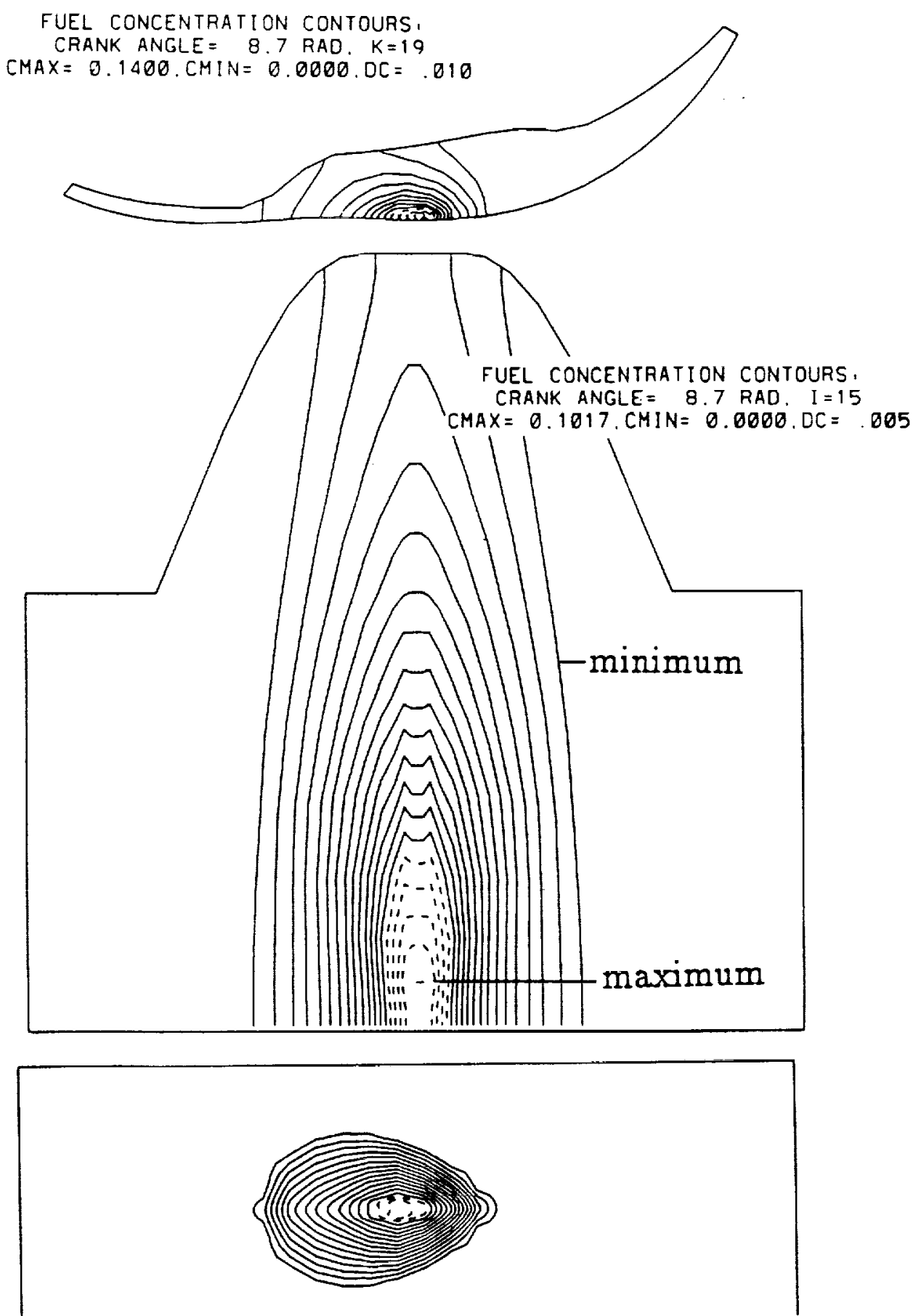

FUEL CONCENTRATION CONTOURS,

CRANK ANGLE $=8.7$ RAD. $J=7$

CMAX $=0.0355$. CMIN $=0.0000 .0 C=.002$

Fig. 10 Fuel mass fraction contours at $\theta=8.75 \mathrm{rad}$. 


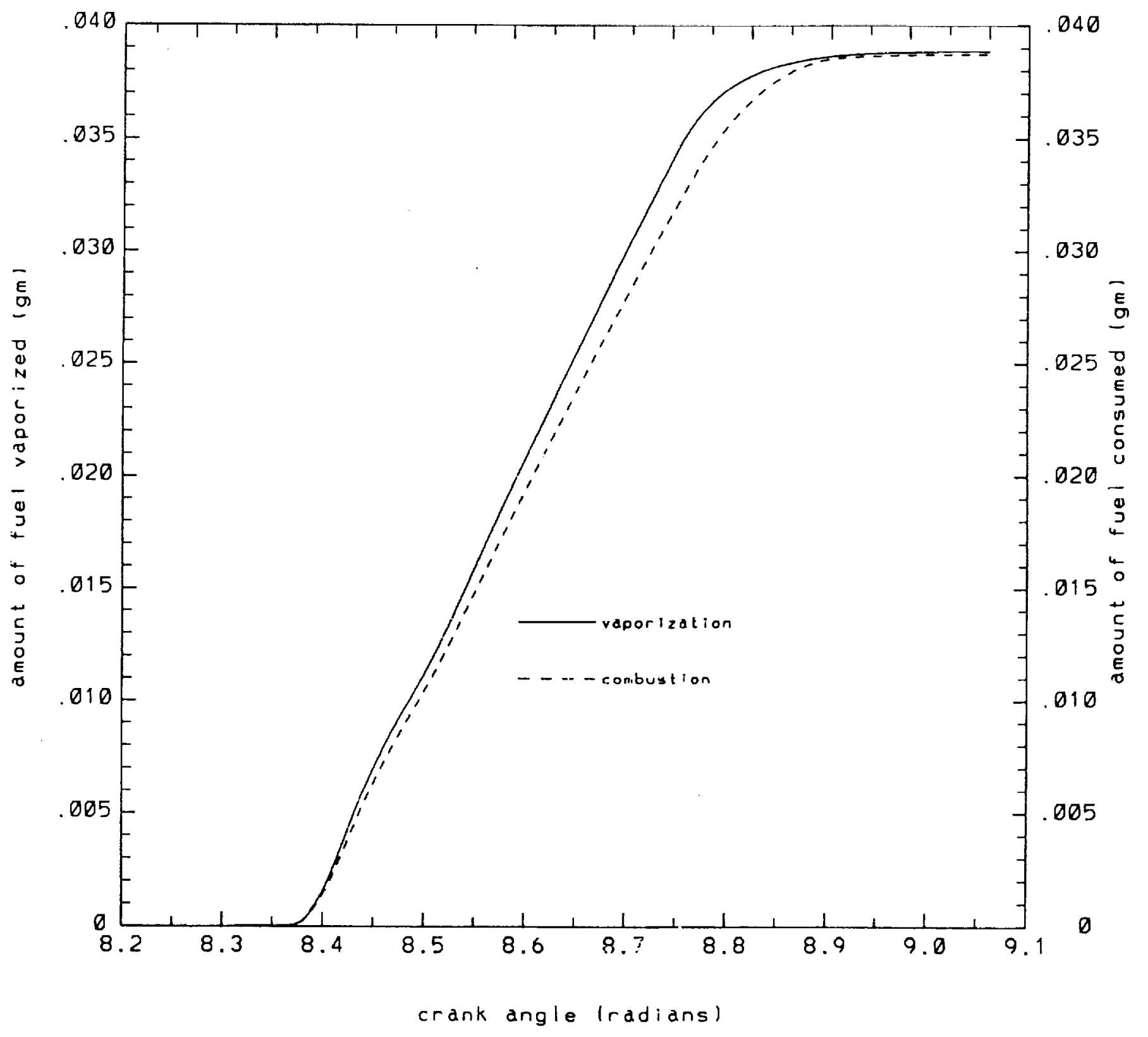

Fig. 11 Variation of amounts of fuel evaporated and reacted versus crank angle. 
VELOCITY VECTOR PLOT,
CRANK ANGLE $=\begin{array}{r}8.3 \mathrm{RAD}, \quad K=19 \\ \text { VMAX }=30.40 \mathrm{M} / \mathrm{S}\end{array}$

(a)

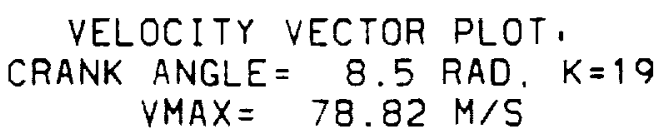

(b)

$$
\begin{aligned}
& \text { ANGLE }=8.5 \mathrm{RAD} . \\
& \text { VMAX }=78.82 \mathrm{M} / \mathrm{S}
\end{aligned}
$$

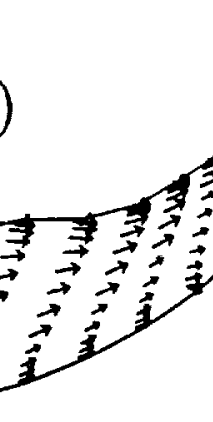

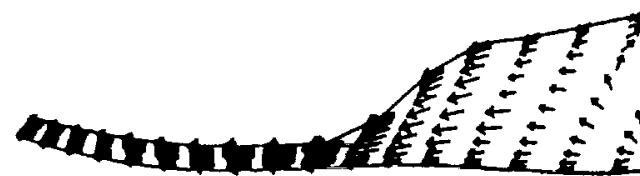

\section{VELOCITY VECTOR PLOT, CRANK ANGLE $=8.7$ RAD. $K=19$ VMAX $=102.61 \mathrm{M} / \mathrm{S}$ \\ (c)}

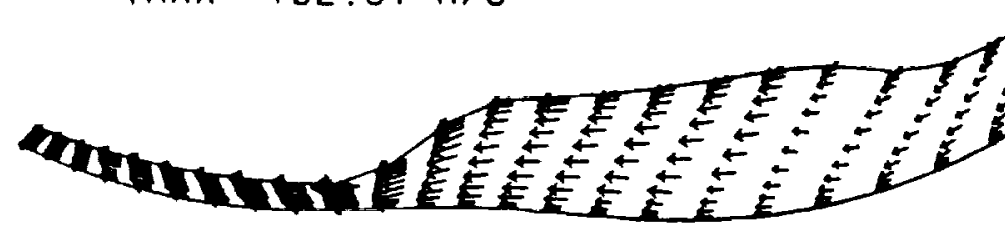
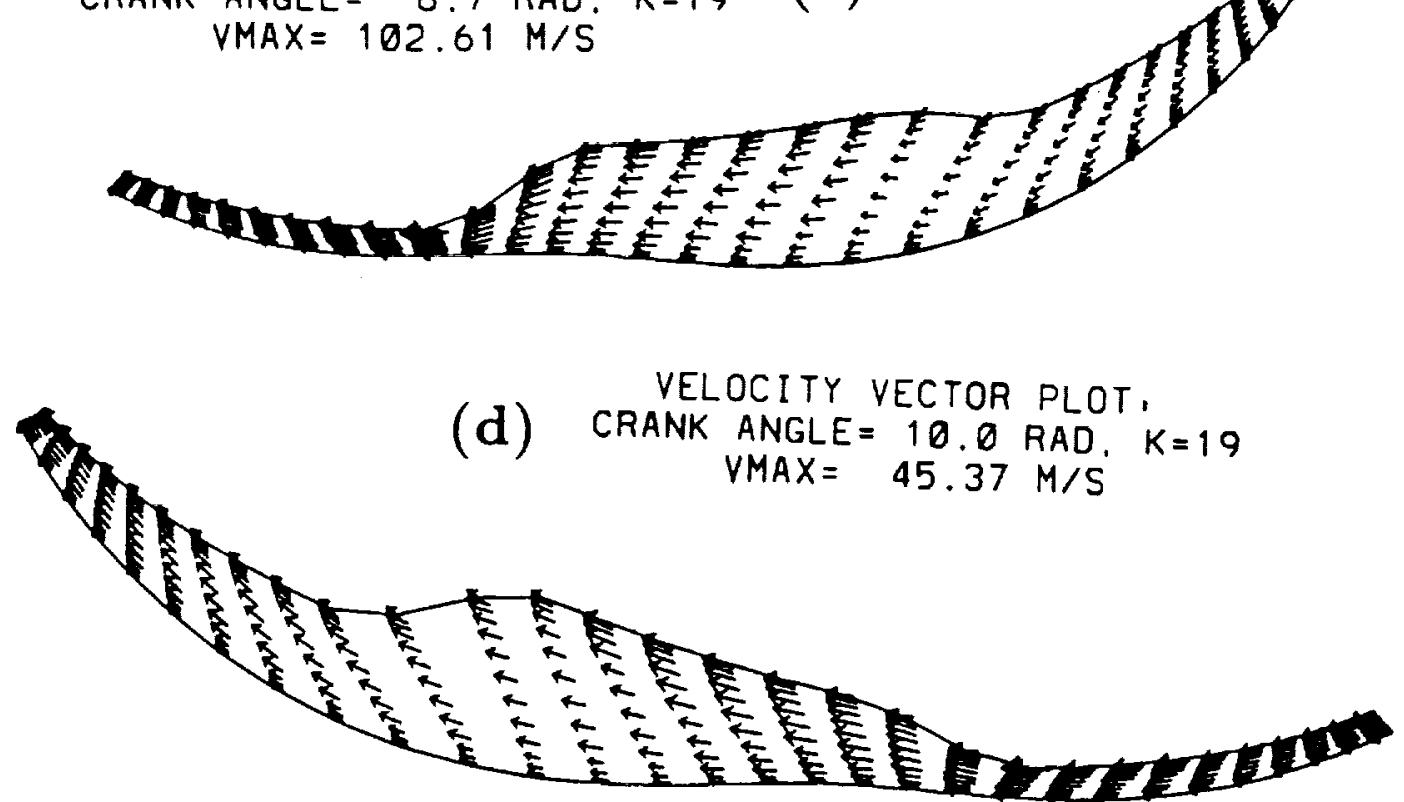

Fig. 12 Velocity vector plots. 
(a) PRESSURE CONTOURS:

CRANK ANGLE $=8.3$ RAD, $K=19$

PMAX $=.57 E+\emptyset 6, P M I N=.48 E+\emptyset 6 \cdot D P=.43 E+\emptyset 4$

minimum

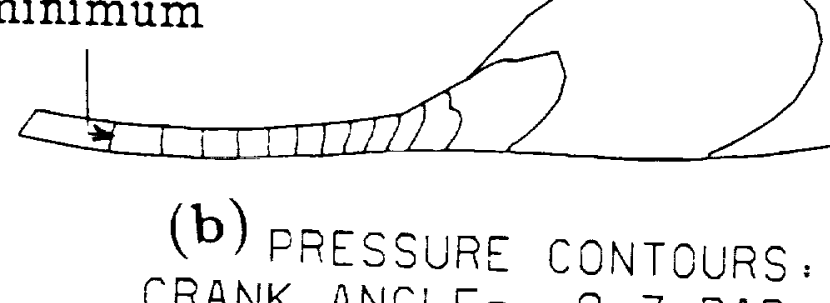

PMAX $=\begin{aligned} & \text { CRANK ANGLE }=8.7 \text { RAD, } \quad K=19 \\ & .36 E+\emptyset 7 . P M I N=.30 E+\square 7, . D P=.45 E+\square 5\end{aligned}$

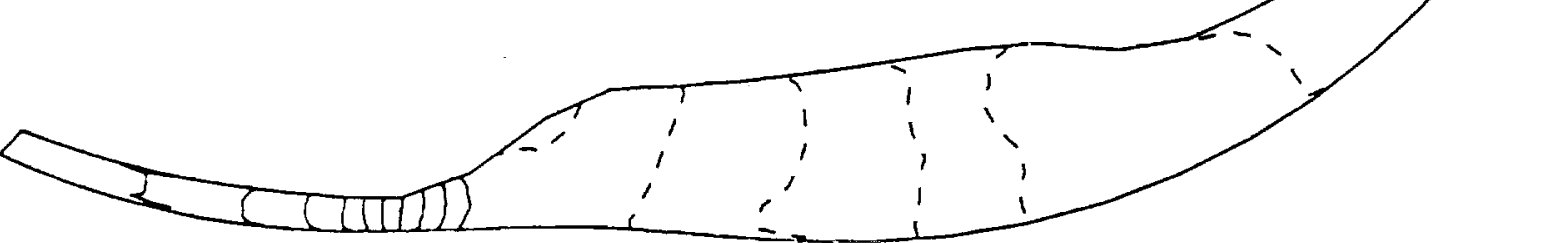

(c) PRESSURE CONTOURS:

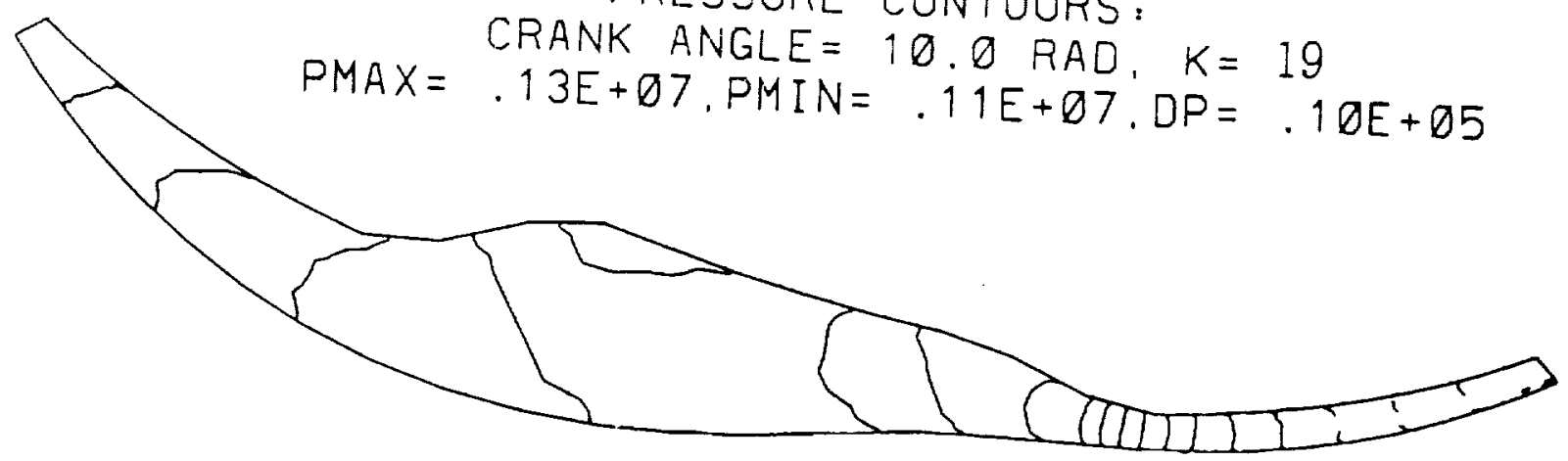

Fig. 13 Pressure contours. 


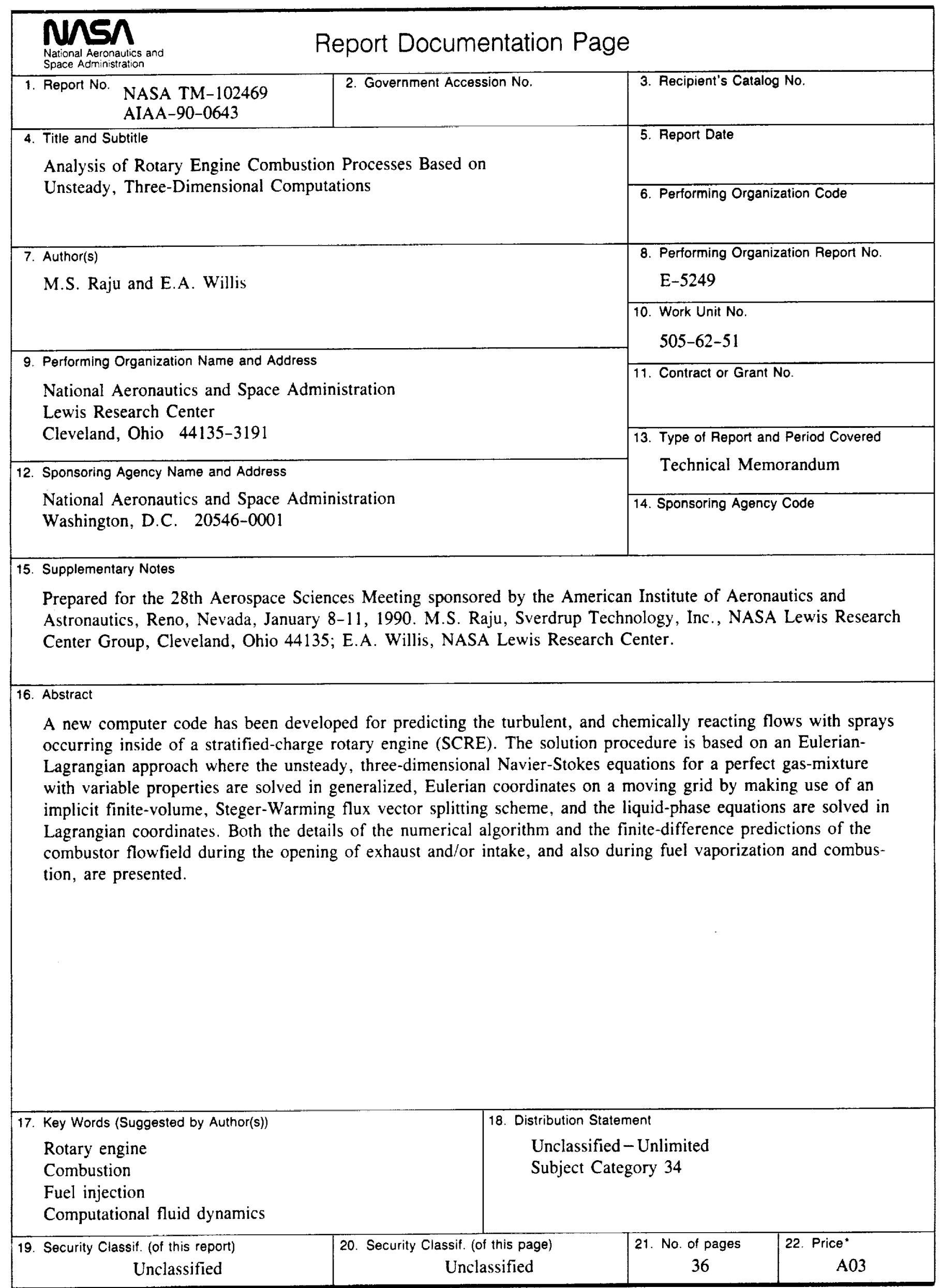

